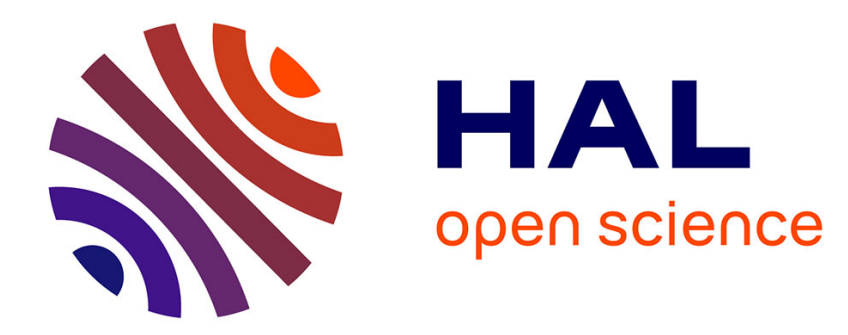

\title{
Uniform controllability of scalar conservation laws in the vanishing viscosity limit \\ Matthieu Léautaud
}

\section{To cite this version:}

Matthieu Léautaud. Uniform controllability of scalar conservation laws in the vanishing viscosity limit. 2010. hal-00512119

\section{HAL Id: hal-00512119 \\ https://hal.science/hal-00512119}

Preprint submitted on 27 Aug 2010

HAL is a multi-disciplinary open access archive for the deposit and dissemination of scientific research documents, whether they are published or not. The documents may come from teaching and research institutions in France or abroad, or from public or private research centers.
L'archive ouverte pluridisciplinaire HAL, est destinée au dépôt et à la diffusion de documents scientifiques de niveau recherche, publiés ou non, émanant des établissements d'enseignement et de recherche français ou étrangers, des laboratoires publics ou privés. 


\title{
Uniform controllability of scalar conservation laws in the vanishing viscosity limit
}

\author{
Matthieu Léautaud ${ }^{* \dagger}$
}

August 27, 2010

\begin{abstract}
We deal with viscous perturbations of scalar conservation laws on a bounded interval with a general flux function $f$ and a small dissipation coefficient $\varepsilon$. Acting on this system on both endpoints of the interval, we prove global exact controllability to constant states with nonzero speed. More precisely, we construct boundary controls so that the solution is driven to the targeted constant state, and we moreover require these controls to be uniformly bounded as $\varepsilon \rightarrow 0^{+}$in an appropriate space. For general (non-convex) flux functions this can be done for sufficiently large time, and for convex fluxes $f$, we have a precise estimate on the minimal time needed to control.
\end{abstract}

\section{Keywords}

Controllability, scalar conservation laws, vanishing viscosity limit.

\section{Contents}

1 Introduction $\quad \mathbf{2}$

1.1 Motivation and main results . . . . . . . . . . . . . . . . . . . . . . . . 2

1.2 Some remarks ... . . . . . . . . . . . . . . . . . . . . . 5

1.3 Structure of the paper, idea of the proofs . . . . . . . . . . . . . . . . 6

2 Three intermediate propositions $\quad \mathbf{8}$

2.1 Approximate controllability using a traveling wave . . . . . . . . . . . . . . . . 8

2.2 Approximate controllability using a rarefaction wave . . . . . . . . . . . . . . . 13

2.3 Local exact controllability . . . . . . . . . . . . . . . . . . . . . . . . . 19

3 Proofs of the three theorems $\quad \mathbf{2 5}$

3.1 Proof of Theorems 1.1 and 1.2 , the convex case . . . . . . . . . . . . . . . 26

3.2 Proof of Theorem 1.3, the non-convex case . . . . . . . . . . . . . . . . 27

4 Appendix: parabolic regularity estimates $\quad \mathbf{2 9}$

4.1 Parabolic regularity estimates for classical solutions . . . . . . . . . . . . . . . . . . 29

4.2 Well-posedness of an initial-boundary value problem with low regularity . . . . . . . 30

${ }^{*}$ Université Pierre et Marie Curie Paris 6, UMR 7598, Laboratoire Jacques-Louis Lions, Paris, F-75005 France; CNRS, UMR 7598 LJLL, Paris, F-75005 France. e-mail: leautaud@ann.jussieu.fr

${ }^{\dagger}$ Laboratoire POEMS, INRIA Paris-Rocquencourt/ENSTA, CNRS UMR 2706, France. 


\section{Introduction}

\subsection{Motivation and main results}

We are concerned with the controllability of the following nonlinear parabolic equation

$$
u_{t}+[f(u)]_{x}-\varepsilon u_{x x}=0 \quad \text { in }(0, T) \times(0, L),
$$

where $T$ is a positive time, $L$ a positive length, and $\varepsilon$ a positive viscosity coefficient. On both endpoints of the interval, we act on the system through the boundary conditions

$$
\left.u\right|_{x=0}=g_{0}(t) \quad \text { in }(0, T),\left.\quad u\right|_{x=L}=g_{L}(t) \quad \text { in }(0, T) .
$$

The problem of exact controllability is the following: given an initial datum

$$
\left.u\right|_{t=0}=u_{0} \quad \text { in }(0, L),
$$

a time $T>0$ and a prescribed state $u_{T}$, is it possible to find control functions $g_{0}=g_{0}^{\varepsilon}$ and $g_{L}=g_{L}^{\varepsilon}$ so that the associated solution $u$ of the system $(1),(2),(3)$ is steered to $u_{T}$ in time $T$ ? The aim of this paper is to prove such a controllability result uniformly with respect to the viscosity coefficient $\varepsilon$ in a sufficiently small range. That is to say, is it possible to find such control functions $g_{0}=g_{0}^{\varepsilon}$, $g_{L}=g_{L}^{\varepsilon}$ which norms in a suitable Banach space remain bounded as $\varepsilon \rightarrow 0^{+}$? More precisely, we shall only consider the uniform controllability to constant targets $u_{T}=M \in \mathbb{R}$. The relevance of this assumption is discussed in Remark 1.5.

Uniform controllability problems for singular perturbations of partial differential equations have already been considered in several works, beginning with [22, Chapter 3]. In the context of transport/heat equation (i.e. $f(u)=V u$ for some constant $V \in \mathbb{R}$ ) in vanishing viscosity limit, this study was initiated by Coron and Guerrero in [9], where the authors make a conjecture on the minimal time needed to achieve uniform controllability. Then, the estimates on this minimal time are improved in [14] with a complex analytic method. The result of [9] was also generalized in several space dimensions and for non-constant transport speed in [19]. Such uniform control properties in singular limits are also addressed for vanishing dispersion in [16] and for vanishing dispersion and viscosity in [17]. All these articles deal with singular perturbations of linear transport equations.

Concerning nonlinear control problems in vanishing viscosity, the only result up to our knowledge has been stated by Glass and Guerrero [15] for the Burgers equation, i.e., in the case $f(u)=\frac{u^{2}}{2}$. Theorems 1.1, 1.2 and 1.3 generalize this for general flux functions.

Our first result is concerned with convex flux functions $f$.

Theorem 1.1. Assume that the flux function satisfies $f \in W_{\mathrm{loc}}^{2, \infty}(\mathbb{R}), f^{\prime \prime} \geq 0$ a.e., and

$$
\begin{gathered}
\liminf _{A \rightarrow+\infty} A^{-\gamma} f^{\prime \prime}(A)>0 \quad ; \quad \limsup _{A \rightarrow+\infty} e^{-A^{2 \gamma+1-\delta}} f^{\prime \prime}(A)<+\infty \text { for some } \gamma>-\frac{1}{2}, \delta>0 \\
\text { (resp. } \left.\liminf _{A \rightarrow-\infty}|A|^{-\gamma} f^{\prime \prime}(A)>0 ; \limsup _{A \rightarrow-\infty} e^{-|A|^{2 \gamma+1-\delta}} f^{\prime \prime}(A)<+\infty \text { for some } \gamma>-\frac{1}{2}, \delta>0\right) .
\end{gathered}
$$

$(\mathrm{A}-)$

Then, there exist $\alpha_{0} \geq 1$ and a constant $C=C\left(\alpha_{0}\right)>0$ such that for all $M$ satisfying $f^{\prime}(M)>0$ (resp. $\left.f^{\prime}(M)<0\right)$, there exists $\varepsilon_{0}>0$ such that for any $u_{0} \in L^{\infty}(0, L)$, any time $T>\alpha_{0} \frac{L}{\left|f^{\prime}(M)\right|}$ and any $\varepsilon \in\left(0, \varepsilon_{0}\right)$, there exist two control functions $g_{0}^{\varepsilon}$ and $g_{L}^{\varepsilon}$ satisfying

$$
\left\|g_{0}^{\varepsilon}\right\|_{L^{\infty}(0, T)}+\left\|g_{L}^{\varepsilon}\right\|_{L^{\infty}(0, T)} \leq C\left(\left\|u_{0}\right\|_{L^{\infty}(0, L)}+|M|\right)
$$

such that the solution of (1), (2) and (3) associated to $g_{0}^{\varepsilon}$ and $g_{L}^{\varepsilon}$ satisfies $\left.u\right|_{t=T}=M$ on $(0, L)$. 
This theorem is the direct generalization of [15, Theorem 1.1]. Note that we shall see that one can take $\alpha_{0}=6$, or, as found numerically $\alpha_{0}=5.3$. Even in the case of a Burgers equation, this improves the minimal control time found in [15] (which is $\alpha_{0}=9$, or, as found numerically $\alpha_{0}=6.3$ ). Having a minimal controllability time is natural here since the inviscid system (for $\varepsilon=0$ ) has a finite propagation speed (see also Remark 1.4 below).

In this theorem, the second part of Assumptions $(\mathrm{A}+)$ and $(\mathrm{A}-)$ is due to a technical argument, and does not seem to be necessary (see also Remarks 2.3 and 2.12 below). Under more natural (and weaker) assumptions, we prove the following (weaker) result for convex flux functions.

Theorem 1.2. Assume that the flux function satisfies $f \in W_{\mathrm{loc}}^{2, \infty}(\mathbb{R}), f^{\prime \prime} \geq 0$ a.e. and $f^{\prime}(u) \rightarrow+\infty$ as $u \rightarrow+\infty$ (resp. $f^{\prime}(u) \rightarrow-\infty$ as $\left.u \rightarrow-\infty\right)$. Then, there exists $\alpha_{0} \geq 1$ and a constant $C=$ $C\left(\alpha_{0}\right)>0$ such that for all $R_{0}>0$ and all $M$ satisfying $f^{\prime}(M)>0\left(\right.$ resp. $\left.f^{\prime}(M)<0\right)$, there exists $\varepsilon_{0}>0$ such that for any $u_{0} \in L^{\infty}(0, L)$ with $\left\|u_{0}\right\|_{L^{\infty}(0, L)} \leq R_{0}$, any time $T>\alpha_{0} \frac{L}{\left|f^{\prime}(M)\right|}$ and any $\varepsilon \in\left(0, \varepsilon_{0}\right)$, there exist two control functions $g_{0}^{\varepsilon}$ and $g_{L}^{\varepsilon}$ satisfying

$$
\left\|g_{0}^{\varepsilon}\right\|_{L^{\infty}(0, T)}+\left\|g_{L}^{\varepsilon}\right\|_{L^{\infty}(0, T)} \leq C\left(R_{0}+|M|\right),
$$

such that the solution of (1), (2) and (3) associated to $g_{0}^{\varepsilon}$ and $g_{L}^{\varepsilon}$ satisfies $\left.u\right|_{t=T}=M$ on $(0, L)$.

The only difference with the result of Theorem 1.1 is that here, $\varepsilon_{0}$ also depends on the norm of the initial datum $u_{0}$. The question whether the result of Theorem 1.1 still holds only with the assumptions of Theorem 1.2 remains open. In particular, Theorem 1.1 does not apply for flux functions satisfying $f(u) \sim_{u \rightarrow+\infty} u^{\rho}$ with $1<\rho \leq \frac{3}{2}$, whereas Theorem 1.2 does.

We also prove a result for non-convex flux functions, that is, for general non linear transport equations. In this setting however, we do not estimate the time needed to control, and our result is less precise. Before stating it, let us define $\sigma(A, B)$ the shock speed between two constant states $A$ and $B$, given by the Rankine-Hugoniot condition, i.e., the slope of the chord of $f$ between $A$ and $B$,

$$
\sigma(A, B)=\frac{f(B)-f(A)}{B-A} \text { if } A \neq B, \quad \text { and } \quad \sigma(A, A)=f^{\prime}(A) .
$$

On the interval $(P, N) \subset \mathbb{R}$, the strict Oleinik admissibility conditions for the flux function $f$ read

$$
\sigma(P, N)<\sigma(A, N), \quad \text { for all } A \in(P, N),
$$

meaning that, on the interval $(P, N)$, the graph of $f$ is below the chord between $P$ and $N$, or

$$
\sigma(P, N)>\sigma(A, N), \quad \text { for all } A \in(P, N), \quad \text { (SOC-) }
$$

meaning that, on the interval $(P, N)$, the graph of $f$ is above the chord between $P$ and $N$. Note that (SOC+) (resp. (SOC-)) is equivalent to having $\sigma(A, P)<\sigma(P, N)($ resp. $\sigma(A, P)\rangle \sigma(P, N))$ for all $A \in(P, N)$. Conditions (SOC+) or (SOC-) ensure the existence of an admissible shock wave between $P$ and $N$ (see [10, Section 8.6]).

We can now state the result concerning non-convex flux functions.

Theorem 1.3. Suppose that $f \in \mathcal{C}^{2}(\mathbb{R})$ and $u_{0} \in L^{\infty}(0, L)$ satisfy the two following conditions:

(i) $\left(f^{\prime \prime}\right)^{-1}(\{0\}) \cap K$ has a finite number of connected components for any compact interval $K \subset \mathbb{R}$,

(ii) There exists an open interval $I \subset \mathbb{R}$ such that $\left[\operatorname{ess} \inf u_{0}\right.$, $\left.\operatorname{ess} \sup u_{0}\right] \subset I$ and $f$ satisfies (SOC + ) or (SOC-) on $I$.

Then, for all $M$ satisfying $f^{\prime}(M) \neq 0$, there exist $C_{0}>0, T_{0}>0$ and $\varepsilon_{0}>0$ (only depending on $I$ and $M)$ such that for any time $T>T_{0}$ and any $\varepsilon \in\left(0, \varepsilon_{0}\right)$, there exist two control functions $g_{0}^{\varepsilon}$ and $g_{L}^{\varepsilon}$ satisfying

$$
\left\|g_{0}^{\varepsilon}\right\|_{L^{\infty}(0, T)}+\left\|g_{L}^{\varepsilon}\right\|_{L^{\infty}(0, T)} \leq C_{0},
$$

such that the solution of (1), (2) and (3) associated to $g_{0}^{\varepsilon}$ and $g_{L}^{\varepsilon}$ satisfies $\left.u\right|_{t=T}=M$ on $(0, L)$. 
Note that Condition $(i)$ actually means that $f^{\prime \prime}$ vanishes on a finite union of points and (closed) intervals on each bounded subset of $\mathbb{R}$. This is generally satisfied except for some pathological examples (for instance $f(u)=u^{5} \cos \left(\frac{1}{u}\right)+u$ if $u \neq 0, f(0)=0$ and the target is 0 ). More precisely, this condition is generically satisfied in $\mathcal{C}^{3}(\mathbb{R})$, since for any compact $K \subset \mathbb{R}$, the set

$$
A=\left\{f \in \mathcal{C}^{3}(K ; \mathbb{R}),\left(f^{\prime \prime}(x), f^{\prime \prime \prime}(x)\right) \neq(0,0) \text { for all } x \in K\right\}
$$

is open and dense in $\mathcal{C}^{3}(K ; \mathbb{R})$. However, note that this genericity property does not hold in the space $\mathcal{C}^{2}(\mathbb{R})($ see $[3])$.

Condition (ii) concerning the initial datum means that there exists an admissible shock wave allowing to get rid of the initial condition, and is more restrictive. For instance, it is not satisfied if $f(u)=\cos (u)$ and $\left[\operatorname{ess} \inf u_{0}\right.$, ess $\sup u_{0}$ ] contains $k \pi$ for some $k \in \mathbb{Z}$. Yet, it is satisfied in a large range of situations, including, for any $u_{0} \in L^{\infty}(0, L)$, the case where $f$ satisfies $\lim _{u \rightarrow+\infty} f^{\prime}(u)= \pm \infty$.

Since we are interested in the properties of uniform controllability as $\varepsilon \rightarrow 0^{+}$, it seems natural to refer to the results that are known both for the viscous problem (for $\varepsilon>0$, fixed) and for the inviscid problem $(\varepsilon=0)$.

First, the controllability of the viscous equation (1) for fixed $\varepsilon>0$ has mainly been considered for the Burgers equation. Two different types of control results have been proved. The local exact controllability to trajectories for this equation has been established in [13]. In this work, the authors also prove that the local exact controllability does not hold as long as one controls in a sub-interval of $(0, L)$, which is equivalent to controlling at one endpoint. Concerning the global exact controllability for the viscous Burgers equation, it is proved in [18], that it does not hold even if the control is acting on both sides of the domain. However, in [7] the author proves a global controllability result from 0 to constant states. More precisely, he states that for $u_{0}=0$ and for any $T>0$, one can drive the solution of (1), (2), (3) to any constant $M$ provided that $|M|$ is sufficiently large with respect to $T$. This result is improved (as a Corollary of the uniform controllability result) in [15], allowing any $u_{0} \in L^{\infty}(0, L)$ and giving a precise condition on the target $M$ and the minimal control time. Finally, adding a third control globally distributed on $(0, L)$, and independent on $x$, the author of [4] establishes the global controllability of the viscous Burgers equation for any $T>0$. Note that this last result is proved by using both controllability properties of the nonviscous equation and a local result.

Second, concerning the inviscid problem

$$
u_{t}+[f(u)]_{x}=0
$$

and in the context of entropy solutions, controllability questions have been addressed by Ancona and Marson for general strictly convex flux functions $f$ in [1]. In this work the controllability problem is posed in the half real line with null initial condition and the set of attainable states is completely described. For the problem on a bounded interval and with a general initial datum, the controllability of the non-viscous Burgers equation $\left(f(u)=\frac{u^{2}}{2}\right)$ was studied in [20], where some conditions are given on the final state in order to ensure this property.

We recall that for conservation laws such as (5), classical solutions starting out from smooth initial data generally develop discontinuities in finite time. As a consequence, only weak solutions may exist for large times. In the context of weak solutions however, uniqueness is lost. To overcome the obstacle of nonuniqueness, restrictions need to be imposed to select the physically relevant weak solution. One criterium for such a selection is to require that the admissible solution satisfies an entropy condition, which reads as follows (see [10, Chapter 6] for instance): for any smooth convex function $\eta: \mathbb{R} \rightarrow \mathbb{R}$ and associated entropy flux $q(u)=\int^{u} \eta^{\prime}(\omega) f^{\prime}(\omega) d \omega$, the following holds in the sense of measures:

$$
\eta(u)_{t}+q(u)_{x} \leq 0 .
$$

Another selection principle is to require that the admissible solution is the limit of a family of solutions of equations containing a diffusive term, such as the one considered here. One can prove (see [21] or $[10$, Chapter 6]) that both definitions coincide, so that entropy solutions are the ones which can be obtained by vanishing viscosity. One can summarize the situation by saying that the viscosity has 
disappeared from the equation, and is only effective for the selection of admissible discontinuities. The Cauchy problem, together with the convergence of vanishing viscosity approximations to the entropy solutions of a general scalar conservation law were studied in the seminal work of Kruzkov $[21]$.

It is therefore very natural, when considering control problems for conservation laws, to study the cost of the viscosity, that is, to determine if known controllability properties for the hyperbolic equation are still valid for the model with small viscosity, and how the size of the control evolves as the viscosity approaches 0 .

Another important motivation for studying singular limit in control problems is the seek of controllability properties for the perturbated system itself. This is well-illustrated by the papers [6], [8] and [5], where the authors invesigate the Navier-Stokes system with Navier slip boundary conditions. They use a global controllability result for the inviscid equation (in this case, the Euler equation) to deduce global approximate controllability of the the Navier-Stokes system. Note that in [6] and [5], global exact controllability is then deduced by a local controllability result obtained by a Carleman inequality. The strategy we use to prove Theorem 1.1 is very close to the one of these works. We here also provide a controllability result for Equation (1) for a fixed viscosity (see Proposition 1.7 below).

Acknowledgements. The author wishes to thank O. Glass and J. Le Rousseau for very fruitful discussions, helpful comments and encouragements, and S. Guerrero for discussions on transposition solutions. The author was partially supported by l'Agence Nationale de la Recherche under grant ANR-09-BLAN-0213-02.

\subsection{Some remarks}

Here, we make some remarks concerning the set of uniformly attainable states, and state two propositions concerning the inviscid system $(\varepsilon=0)$ and the viscous system with $\varepsilon=1$.

Remark 1.4. In general, entropy solutions of (5) cannot reach a state $u_{T}$ (starting for instance from $\left.u_{0}=0\right)$, in a time less than $\frac{L}{\inf \left|f^{\prime}\left(u_{T}\right)\right|}$. In particular, the states $u_{T}$ satisfying $f^{\prime}\left(u_{T}\right)=0$ cannot be reached unless one has $u_{0}=u_{T}$. This can be proved by considering generalized backward characteristics (see [1]). Hence the minimal control time $\alpha_{0} \frac{L}{\left|f^{\prime}(M)\right|}$ in Theorem 1.1 is not surprising. Note that even in the cases of linear transport equation at speed $M \in \mathbb{R}$ or Burgers equation, the uniform controllability results [9], [14], [17], [19] and [15] consider a time of control of the form $C \frac{L}{|M|}$, $C>1$.

Remark 1.5. Here, we are looking for the set $\mathcal{E}_{T}$ of states that are controllable uniformly in the asymptotics $\varepsilon \rightarrow 0^{+}$at time $t=T$. This implies in particular that $\mathcal{E}_{T}$ is contained in the reachable set $\mathcal{E}_{T}^{0}$ for the nonviscous equation (5) and in the reachable set $\mathcal{E}_{T}^{\varepsilon}$ for the viscous equation (1), for any $\varepsilon \in\left(0, \varepsilon_{0}\right): \mathcal{E}_{T} \subset \bigcap_{0<\varepsilon<\varepsilon_{0}} \mathcal{E}_{T}^{\varepsilon} \cap \mathcal{E}_{T}^{0}$. In general, this intersection seems difficult to describe since the solutions of (1) are very regular whereas the solutions of (1) can have discontinuities. We thus restrict ourselves to equilibrium points of the system, that are the most interesting states to control. Let $u_{T}(x)$ be a uniformly controllable stationary state as $\varepsilon \rightarrow 0^{+}$. It satisfies both

$$
f\left(u_{T}\right)_{x}=0, \quad \text { and } \quad f\left(u_{T}\right)_{x}-\varepsilon u_{T, x x}=0, \quad \text { on }(0, L),
$$

so that we have, for some constants $c$ and $d$,

$$
u_{T}(x)=c x+d, \quad \text { and } \quad f^{\prime}(c x+d) c=0, \quad \text { on }(0, L) .
$$

As a consequence, either $c=0$ and $u_{T}$ is constant, or $c \neq 0, u_{T}(x)=c x+d$ and $f^{\prime}\left(u_{T}\right)=0$ on $(0, L)$. Referring to Remark 1.4, we see that states satisfying $f^{\prime}\left(u_{T}\right)=0$ are not attainable for the inviscid system (5), and necessarily $u_{T}=d$ is a constant. Finally, the set of uniformly attainable stationary states for (1) is exactly the set of constant states with non-zero speed. 
In the vanishing viscosity limit $\varepsilon \rightarrow 0^{+}$, (the proof of) Theorem 1.3 gives a controllability result to constant states for entropy solutions of (5), which is new as well.

Proposition 1.6. Suppose that $f \in \mathcal{C}^{2}(\mathbb{R})$ and $u_{0} \in L^{\infty}(0, L)$ satisfy conditions $(i)$ and (ii) of Theorem 1.3. Then, for all $M$ satisfying $f^{\prime}(M) \neq 0$, there exists $T_{0}>0$ and an entropy solution $u \in \mathcal{C}^{0}\left(\mathbb{R}_{+} ; L_{\text {loc }}^{1}(\mathbb{R})\right)$ of

$$
u_{t}+[f(u)]_{x}=0 \quad \text { in } \mathbb{R}_{+} \times \mathbb{R},
$$

such that

$$
\left.u\right|_{t=0}=u_{0} \quad \text { and }\left.\quad u\right|_{t=T}=M \quad \text { on }(0, L),
$$

for any time $T>T_{0}$.

Even if this proposition can be viewed as a consequence of Theorem 1.3, it can also be proved directly constructing entropy solutions. Such a proof follows the construction of Section 3.2, using shock waves in place of traveling waves and rarefaction waves in place of viscous ones. This type of direct proof of Proposition 1.6 would already contain all the ideas and the difficulties of Theorem 1.3 since the vanishing viscosity problem is addressed separately in Section 2 .

Note that this proposition can be seen as a boundary control result for conservation laws on the interval $(0, L)$. However, in this case, one has to take care of the sense we give to boundary conditions. Indeed, they must not be understood in the sense of Dirichlet, which is not the adapted notion for conservation laws, but rather in the sense of [2] or [1].

Note also that even in the case of a constant viscosity, the analogous of Theorem 1.3 is a new global controllability result to constant states in large time for semilinear heat equations. More precisely, we have

Proposition 1.7. Suppose that $f \in \mathcal{C}^{2}(\mathbb{R})$ and $u_{0} \in L^{\infty}(0, L)$ satisfy conditions $(i)$ and $(i i)$ of Theorem 1.3. Then, for all $M$ satisfying $f^{\prime}(M) \neq 0$, there exists $T_{0}>0$ such that for any time $T>T_{0}$, there exist two control functions $g_{0}, g_{L} \in L^{\infty}(0, T)$ such that the solution of

$$
\begin{cases}u_{t}-u_{x x}+[f(u)]_{x}=0 & \text { in }(0, T) \times(0, L), \\ \left.u\right|_{x=0}=g_{0}(t) \text { and }\left.u\right|_{x=L}=g_{L}(t) & \text { in }(0, T), \\ \left.u\right|_{t=0}=u_{0} & \text { in }(0, L),\end{cases}
$$

satisfies $\left.u\right|_{t=T}=M$ in $(0, L)$.

To prove this proposition, it suffices to follow the proof of Theorem 1.3 line by line and replace the argument " $\varepsilon$ small" by " $T$ large". It works since all the constants we obtain in the approximate controllability arguments are of the form $e^{-K \frac{T}{\varepsilon}}$ (see also Remark 2.7 below).

\subsection{Structure of the paper, idea of the proofs}

The main idea for proving Theorems 1.1, 1.2 and 1.3 is to combine global approximate controllability results relying on the hyperbolic nature of the problem and local exact controllability relying on the parabolic perturbation term.

The proof of Theorems 1.1 and 1.2 follows the strategy of the article [15]. One of its main ingredients is the use of the return method of J.-M. Coron, which consists in finding a particular trajectory of the system which moves far away from the initial state to get back to the final state afterwards. This strategy to prove global controllability results is for instance very close to the one used in [6], [8] and [5] for the Navier Stokes equations and [4] for the Burgers equation. In the situation of Theorems 1.1 and 1.2, we steer the system to a large constant state $N$ (such that $f^{\prime}(N)$ has the same sign as $f^{\prime}(M)$ ), and then we get back to the constant target $M$. The first step (reaching $N)$ can be done as fast as needed, taking $N$ sufficiently large.

The main difference between our proof of Theorems 1.1, 1.2 and the one in [15] is concerned with the global approximate controllability results (see Sections 2.1 and 2.2). The proofs given in [15] for the Burgers equation rely on the Hopf formula, which gives an explicit expression of the solution 
of the viscous Burgers equation on the real line. Up to our knowledge, this formula does not exist for general flux functions, so we have to develop different arguments. We hence have to study the convergence rate of viscous shock waves (resp. viscous rarefaction waves) to non viscous ones as $\varepsilon \rightarrow 0^{+}$.

Then, the proof of Theorem 1.3 relies on an iterative version of proof of Theorem 1.2. In a first step, we use Assumption ( $i i$ ) to get rid of the initial condition thanks to a viscous shock wave (see a definition in the next paragraph). After that, under Assumption $(i)$, there is between the initial datum and the target a finite number of zones on which $f^{\prime \prime} \geq 0$ or $f^{\prime \prime} \leq 0$. In each of these intervals we can develop the same type of arguments as in the proof of Theorem 1.2. We also have to study how to pass from one zone to another. Note that in this proof, the difficulty does not come from the uniformity with respect to $\varepsilon$. The problem here is to handle the non monotonicity of the speeds (i.e. the non-convex flux function), and is hence of hyperbolic nature. The inviscid framework (for $\varepsilon=0$ ) already contains the difficulties encountered here, and the proof still holds in this case (see Proposition 1.6 above)

The ouline of the paper is the following: In Section 2, we prove three intermediate propositions needed for the proof of Theorems 1.1, 1.2 and 1.3. Sections 2.1 and 2.2 are concerned with two different global approximate controllability results. Then, Section 2.3 deals with the local exact controllability argument, that will be used systematically after the approximate controllability results of both Sections 2.1 and 2.2. In Section 3, we finally combine these arguments and conclude the proofs of Theorems 1.1, 1.2 and 1.3. Note that the local exact controllability result is proved using a fixed point argument for which we need to have a small parameter ( $\varepsilon$ in the three theorems) or a large parameter ( $T$ in Proposition 1.7 or $N$ in the first step of the proof of Theorem 1.1).

To conclude this section, let us introduce the traveling wave (or viscous shock wave) solutions of (1), and recall some of their basic properties (see [10, Section 8.6]). In the following, we shall make an intensive use of these solutions. Searching a solution $\check{u}$ of (1) under the form

$$
\check{u}(t, x)=U\left(\frac{x-s t}{\varepsilon}\right), \quad(t, x) \in \mathbb{R}_{+} \times \mathbb{R},
$$

that approximates as $\varepsilon \rightarrow 0^{+}$a shock wave between $N$ and $P$, leads to considering the ordinary differential equation

$$
\begin{gathered}
\dot{U}=f(U)-f(N)-s(U-N), \\
s=\sigma(P, N), \\
\lim _{\xi \rightarrow+\infty} U(\xi)=P, \quad \text { and } \lim _{\xi \rightarrow-\infty} U(\xi)=N,
\end{gathered}
$$

once having replaced $\check{u}$ by $U$ in (1) and integrated. Here $\dot{U}$ denotes the derivative with respect to $\xi$, $U=U(\xi), \xi \in \mathbb{R}$ is the wave profile and $s$ the speed of the wave. This speed is exactly the speed of the associated shock wave and is prescribed by the Rankine-Hugoniot condition (7). Under the assumptions (SOC+) and $P<N$ (resp. (SOC-) and $P>N$ ), System (6)-(8) admits a solution (see [10, Section 8.6]), that moreover has the following properties:

- $U$ is decreasing (resp. increasing) from $N$ to $P$, since the Rankine-Hugoniot condition (7) together with the fact that $P<U(\xi)<N$ (resp. $N<U(\xi)<P$ ) for all $\xi \in \mathbb{R}$ implies that the vector field in the right hand-side of (6) is always negative (resp. positive);

- $\lim _{\xi \rightarrow \pm \infty} \dot{U}(\xi)=0$, as a consequence of (6)-(8);

- for any $\xi_{0} \in \mathbb{R}, U_{\xi_{0}}=U\left(\cdot-\xi_{0}\right)$ is still a solution of (6)-(8) since (6) is autonomous;

- $U \in W^{3, \infty}(\mathbb{R})$ for $f \in W_{\text {loc }}^{2, \infty}(\mathbb{R})$ by a bootstrap argument in $(6)$. 
In the following, we shall say that a solution $U$ of (6)-(8) is a traveling wave "from $P$ to $N$ " if the vector field in the right hand side of (6) is oriented from $P$ to $N$. If (SOC+) is satisfied between $P$ and $N, U$ is a traveling wave "from $P$ to $N$ " if $P<N$ and $s>0$ or if $P>N$ and $s<0$. If (SOC-) is satisfied between $P$ and $N, U$ is a traveling wave "from $P$ to $N$ " if $P<N$ and $s<0$ or if $P>N$ and $s>0$.

Remark 1.8. In the sequel, during proofs, $C$ will denote a generic positive constant, whose value may change from line to line. Writing $C=C(p, \beta, \ldots)$ means that this constant depends on the parameters $p, \beta, \ldots$.

\section{Three intermediate propositions}

\subsection{Approximate controllability using a traveling wave}

Here, we prove the approximate controllability to a large state $N$, using a traveling wave. Given $A, B \in \mathbb{R}$, let us recall that $\sigma(A, B)$ is defined in (4) and denotes the slope of the chord of $f$ between $A$ and $B$. It represents the speed of the potential shock wave between $A$ and $B$, if it is admissible. Since $f \in W_{\text {loc }}^{2, \infty}(\mathbb{R})$, we have $\sigma \in W_{\text {loc }}^{1, \infty}\left(\mathbb{R}^{2}\right)$. On the interval $(P, N] \subset \mathbb{R}$, we shall use the following particular version of the Oleinik condition for the flux function $f$, which is a sufficient admissibility condition for a shock wave betwen $P$ and $N$ :

$$
\sigma(P, N)<\sigma(A, N), \quad \text { for all } A \in(P, N] \text {. }
$$

This is the strict Oleinik condition (SOC+) with the additional assumption $\sigma(P, N)<f^{\prime}(N)$. Note that (POC) implies in particular the existence of a traveling wave (with speed $\sigma(P, N)$ ) between $P$ and $N$ (which is equivalent to the usual non-strict Oleinik condition, see [10, Section 8.6]). Under the aditional assumption $f(N)>f(P)$, the speed of this traveling wave is positive. According to the convention described at the end of Section 1.3, it is a traveling wave from $P$ to $N$.

Proposition 2.1. Suppose that there exist constant states $P$ and $N$, with $P<\operatorname{ess} \inf u_{0} \leq \operatorname{ess} \sup u_{0}<$ $N$, such that (POC) holds on $(P, N]$, and $f(N)>f(P)$. Then, for all $\varepsilon>0$, there exist control functions $g_{0}$ and $g_{L}$ with

$$
\left\|g_{0}\right\|_{L^{\infty}(0,+\infty)} \leq \max (|N|,|P|) \quad \text { and } \quad\left\|g_{L}\right\|_{L^{\infty}(0,+\infty)} \leq \max (|N|,|P|),
$$

such that the solution $u$ of (1), (2), (3) satisfies, for any $\kappa>0, \nu \in(0,1)$ and $t^{*}>\frac{L+\kappa}{(1-\nu) s}$,

$$
\begin{aligned}
\left\|u\left(t^{*}, \cdot\right)-N\right\|_{H^{1}(0, L)} \leq C_{\kappa} \frac{t^{* 2}}{\varepsilon^{\frac{5}{2}}}(N-P)\left\|f^{\prime}\right\|_{L^{\infty}(P, N)}^{\frac{5}{2}} & \\
& \left(\left\|f^{\prime}\right\|_{L^{\infty}(P, N)}+\left\|f^{\prime \prime}\right\|_{L^{\infty}(P, N)}\|f\|_{L^{\infty}(P, N)}\right) e^{-\frac{\nu}{\varepsilon} s\left(\sigma_{m}-s\right) t^{*}},
\end{aligned}
$$

where $s=\sigma(P, N), \sigma_{m}=\min \left\{\sigma(A, N), A \in\left[\operatorname{ess} \inf u_{0}, N\right]\right\}$ and $C_{\kappa}$ is independent from $t^{*}, P, N, \varepsilon$.

Note that $s>0$ and $\sigma_{m}>s$ under Assumptions (POC) and $f(N)>f(P)$, so that $u$ converges exponentially to $N$ on the interval considered as $\varepsilon \rightarrow 0^{+}$or $t^{*} \rightarrow+\infty$. In the case where $f$ is convex, we have $\sigma_{m}=\sigma\left(\operatorname{ess} \inf u_{0}, N\right)$ since $\sigma(\cdot, N)$ is nondecreasing. If we replace (POC) by (SOC+) in the assumptions of Proposition 2.1, then $\sigma_{m}=\inf \left\{\sigma(A, N), A \in\left[\operatorname{ess} \inf u_{0}, N\right)\right\}$, and $\sigma_{m}=s$ can occur. Under the weaker assumption ( $\mathrm{SOC}+$ ), we thus no longer have systematically exponential decay.

Proof of Proposition 2.1. The solution $u$ in Proposition 2.1 is obtained by taking the restriction to $\left(0, t^{*}\right) \times(0, L)$ of the solution defined on whole $\mathbb{R}_{+} \times \mathbb{R}$ (still denoted $u$ ) of the following problem (see Figure 1)

$$
\begin{cases}u_{t}+[f(u)]_{x}-\varepsilon u_{x x}=0 & \text { in } \mathbb{R}_{+} \times \mathbb{R} \\ \left.u\right|_{t=0}=\tilde{u}_{0} & \text { in } \mathbb{R}\end{cases}
$$


with

$$
\tilde{u}_{0}(x)=\left\{\begin{array}{l}
N \quad \text { if } x \leq 0, \\
u_{0} \quad \text { if } x \in(0, L), \\
\text { ess inf } u_{0} \quad \text { if } x \geq L .
\end{array}\right.
$$

Then, the control functions $g_{0}$ and $g_{L}$ are obtained by taking the trace of $u$ along the lines $\left(0, t^{*}\right) \times\{0\}$ and $\left(0, t^{*}\right) \times\{L\}$. The estimates on the control cost (9) follow directly from the comparison principle [10, Theorem 6.3.2], and hence, we only have to prove (10). We first prove the following lemma, that gives a $L^{\infty}$ estimate of the convergence rate of traveling waves (viscous shock waves) to the associated non-viscous shock wave as $\varepsilon \rightarrow 0^{+}$. Then a comparison principle gives a $L^{\infty}$ estimate of the convergence rate of $u$ to $N$. This strategy is illustrated in Figure 1.
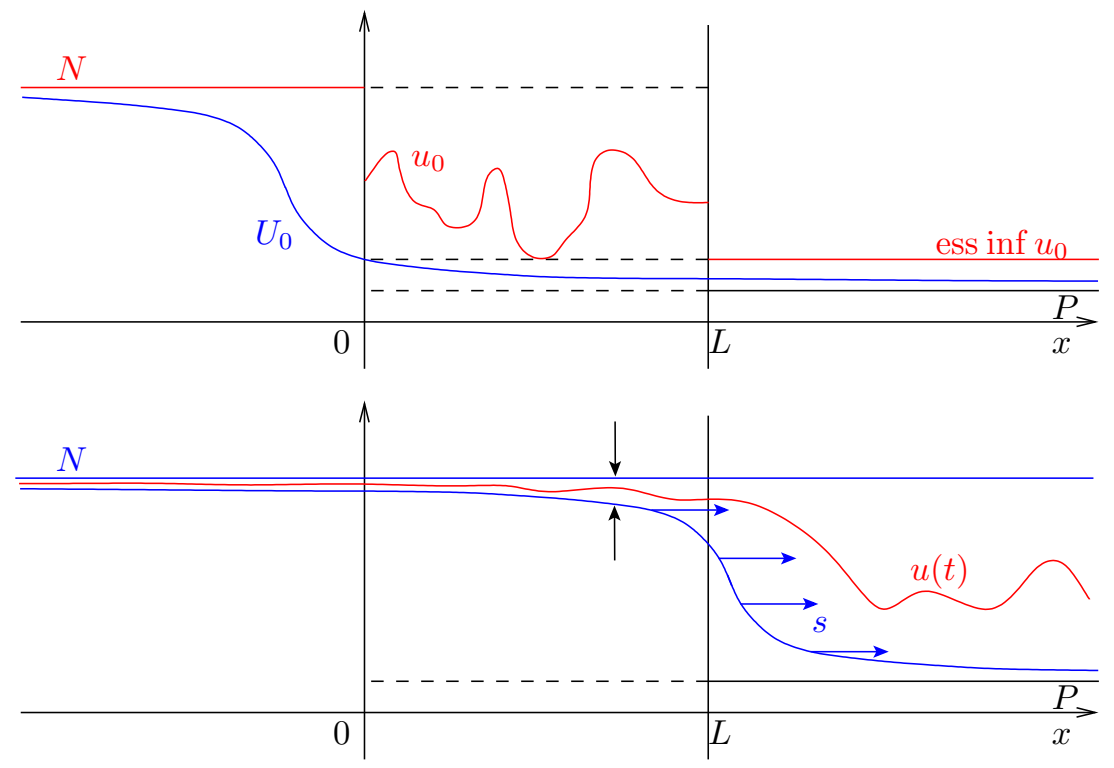

Figure 1: Comparison principle during the traveling wave. Initial data, and solution after a time $t>\frac{L}{s}$ has gone by.

Lemma 2.2. Suppose that there exist constant states $P$ and $N$, with $P<\operatorname{ess}$ inf $u_{0} \leq \operatorname{ess} \sup u_{0}<N$, such that (POC) holds on $(P, N]$, and $f(N)>f(P)$. Then, for every $\varepsilon>0$ and $\beta \in \mathbb{R}$, the solution $u$ of (11), (12) satisfies

$$
\|N-u(t, \cdot)\|_{L^{\infty}(-\infty, \beta)} \leq\left(N-\operatorname{ess} \inf u_{0}\right) e^{-\frac{s}{\varepsilon}\left(\sigma_{m}-s\right)\left(t-\frac{\beta}{s}\right)}, \quad \text { for } t>\frac{\beta}{s},
$$

where $s=\sigma(P, N)$ and $\sigma_{m}=\min \left\{\sigma(A, N), A \in\left[\operatorname{ess} \inf u_{0}, N\right]\right\}$.

Proof. We introduce the family of traveling waves from $P$ to $N$, given by

$$
\check{u}(t, x)=U\left(\frac{x-s t}{\varepsilon}\right), \quad(t, x) \in \mathbb{R}_{+} \times \mathbb{R}
$$

whose existence is ensured by Assumption (POC) (see [10, Section 8.6]). Here, $U=U(\xi), \xi \in \mathbb{R}$ is the wave profile and $s=\sigma(P, N)>0$ the speed of the wave. The ordinary differential equation (6) satisfied by $U$ can be reformulated as

$$
\frac{d}{d \xi}(U-N)=(-s+\sigma(U, N))(U-N)
$$


which yields, for any $\xi, \xi_{0} \in \mathbb{R}$

$$
(U(\xi)-N)=\left(U\left(\xi_{0}\right)-N\right) \exp \left(\int_{\xi_{0}}^{\xi}(-s+\sigma(U(\tau), N)) d \tau\right) .
$$

From now on, we select the traveling wave that satisfies

$$
U_{0}(0)=\operatorname{ess} \inf u_{0}>P,
$$

(which is unique since it now solves a Cauchy problem) so that

$$
0<N-U_{0}(\xi)=\left(N-\operatorname{ess} \inf u_{0}\right) \exp \left(\int_{0}^{\xi}\left(-s+\sigma\left(U_{0}(\tau), N\right)\right) d \tau\right) .
$$

Note also that $U(\xi) \rightarrow P$ when $\xi \rightarrow+\infty$, and the solution $U_{0}$ can also be characterized by

$$
\int_{0}^{+\infty}\left(-s+\sigma\left(U_{0}(\tau), N\right)\right) d \tau=\log \left(\frac{N-P}{N-\operatorname{essinf} u_{0}}\right) .
$$

For $\xi<0$ and $\tau \in[\xi, 0]$, we have $U_{0}(\tau) \in\left[U_{0}(0), U_{0}(\xi)\right] \subset\left[U_{0}(0), N\right]$. On the compact interval $\left[U_{0}(0), N\right]$, the function $A \mapsto \sigma(A, N)$ is continuous and satisfies $\sigma(A, N)>s$, so that

$$
-s+\sigma\left(U_{0}(\tau), N\right) \geq \sigma_{m}-s>0, \quad \text { for all } \xi<0, \tau \in[\xi, 0] .
$$

As a consequence,

$$
\int_{0}^{\xi}-s+\sigma\left(U_{0}(\tau), N\right) d \tau \leq \xi\left(\sigma_{m}-s\right), \quad \text { for all } \xi<0,
$$

so that (14) now yields

$$
0<N-U_{0}(\xi) \leq\left(N-\operatorname{essinf} u_{0}\right) e^{\xi\left(\sigma_{m}-s\right)}, \quad \text { for all } \xi<0 .
$$

Coming back to the variables $t, x$, we obtain for $x \in(-\infty, \beta)$

$$
0<N-U_{0}\left(\frac{x-s t}{\varepsilon}\right) \leq\left(N-\operatorname{ess} \inf u_{0}\right) e^{\frac{1}{\varepsilon}\left(\sigma_{m}-s\right)(\beta-s t)}, \quad \text { for } t>\frac{\beta}{s},
$$

since $U_{0}$ is decreasing. We conclude the proof by comparing the traveling wave $U_{0}$ and the solution $u$ of (11), (12). From the choice of $U_{0}(0)$, we have at time $t=0$,

$$
U_{0}\left(\frac{x}{\varepsilon}\right) \leq \tilde{u}_{0}(x) \leq N, \quad \text { for all } x \in \mathbb{R}, \varepsilon>0 .
$$

The comparison principle $\left[10\right.$, Theorem 6.3.2] then implies that for every $(t, x) \in \mathbb{R}_{+} \times \mathbb{R}$,

$$
U_{0}\left(\frac{x-s t}{\varepsilon}\right) \leq u(t, x) \leq N,
$$

so that estimate (15) now yields

$$
\|N-u(t, \cdot)\|_{L^{\infty}(-\infty, \beta)} \leq\left(N-\operatorname{ess} \inf u_{0}\right) e^{-\frac{s}{\varepsilon}\left(\sigma_{m}-s\right)\left(t-\frac{\beta}{s}\right)}, \quad \text { for } t>\frac{\beta}{s},
$$

and the lemma is proved.

Remark 2.3. If $f$ is convex on $(P, N)$, the decay rate obtained does not seem to be optimal. In this case, under the assumptions of Lemma 2.2, one can prove that for any $\theta<1$, there exists $\xi_{0}=\xi_{0}(N)>0$ and $C>0$ such that

$$
\|N-u(t, \cdot)\|_{L^{\infty}(-\infty, \beta)} \leq C e^{-\frac{\theta}{\varepsilon} s\left(f^{\prime}(N)-s\right)\left(t-\frac{\beta}{s}\right)}, \quad \text { for } t>\frac{\beta+\xi_{0} \varepsilon}{s} .
$$


The convergence rate that we can expect is thus in the convex case of the form $\theta s\left(f^{\prime}(N)-s\right)$. This plays an important role when taking $N$ large, as in the first part of the proof of Theorem 1.1 since $\theta s\left(f^{\prime}(N)-s\right)$ is much larger than $s\left(\sigma_{m}-s\right)$. For the Burgers equation [15] for instance, $\theta s\left(f^{\prime}(N)-s\right) \sim \theta N^{2} / 4$ whereas $s\left(\sigma_{m}-s\right) \sim\left(\right.$ ess inf $\left.u_{0}-P\right) N$ as $N \rightarrow+\infty$. The problem for general convex functions $f$ is to give the asymptotic behaviour of $\xi_{0}(N)$. If $\xi_{0}(N) / s \rightarrow 0$, then Assumption $(\mathrm{A}+)$ can be replaced by the more general (and somehow more natural) assumption:

$$
\liminf _{A \rightarrow+\infty} A^{-\gamma} f^{\prime \prime}(A)>0 \quad ; \quad \limsup _{A \rightarrow+\infty} e^{-A^{2 \gamma+2-\delta}} f^{\prime \prime}(A)<+\infty \text { for some } \gamma>-1, \delta>0
$$

Such a condition would include in particular flux functions satisfying $f(u) \sim_{u \rightarrow+\infty} u^{\rho}$ with $1<\rho \leq \frac{3}{2}$, for which Assumption $(\mathrm{A}+)$ does not hold.

We can now conclude the proof of Proposition 2.1 by a bootstrap argument.

Back to proof of Proposition 2.1. We study the evolution of $(11),(12)$ for $t \in\left(0, t^{*}\right)$, where $t^{*}$ is the time at which we want to obtain Estimate (10). First, we set

$$
v(t, x)=(u-N)\left(t, x+f^{\prime}(N) t\right)
$$

so that, for any $\beta \in \mathbb{R}$,

$$
\|v(t, \cdot)\|_{L^{\infty}(-\infty, \beta)}=\|u(t, \cdot)-N\|_{L^{\infty}\left(-\infty, \beta+f^{\prime}(N) t\right)} .
$$

Estimate (13) of Lemma 2.2 gives,

$$
\|v(t, \cdot)\|_{L^{\infty}(-\infty, \beta)} \leq\left(N-\operatorname{ess} \inf u_{0}\right) \exp \left(-\frac{s}{\varepsilon}\left(\sigma_{m}-s\right)\left(t-\frac{\beta+f^{\prime}(N) t}{s}\right)\right),
$$

for $t>\frac{\beta+f^{\prime}(N) t}{s}$, i.e. $t<\frac{-\beta}{f^{\prime}(N)-s}$. For some $\mu>1$ that will be chosen later on, we fix $\beta^{*}=$ $-\left(f^{\prime}(N)-s\right) \mu t^{*}<0$, so that estimate (16) holds for every $t \in\left(0, \mu t^{*}\right)$. Estimate (16) now becomes

$$
\|v(t, \cdot)\|_{L^{\infty}\left(-\infty, \beta^{*}\right)} \leq\left(N-\operatorname{ess} \inf u_{0}\right) \exp \left(-\frac{1}{\varepsilon}\left(\sigma_{m}-s\right)\left(f^{\prime}(N)-s\right)\left(\mu t^{*}-t\right)\right), \quad \text { for } t<\mu t^{*}
$$

We denote by $\Omega_{1}=\left(a_{1}, b_{1}\right)$ a bounded open interval of $\left(-\infty, \beta^{*}\right)$ and $\chi_{1} \in \mathcal{C}_{c}^{\infty}\left(\Omega_{1}\right)$ a cut-off function satisfying $\chi_{1}=1$ on some $\Omega_{2}$, with $\bar{\Omega}_{2} \subset \Omega_{1}$. The function $w_{1}(t, x)=\chi_{1}(x) v(t, x)$ satisfies

$$
\begin{cases}w_{1, t}-\varepsilon w_{1, x x}=\left[f^{\prime}(N)-f^{\prime}(u)\right]\left(w_{1, x}-\chi_{1}^{\prime} v\right)-\varepsilon\left(\chi_{1}^{\prime \prime} v+2 \chi_{1}^{\prime} v_{x}\right) & \text { in }\left(0, t^{*}\right) \times \Omega_{1} \\ w_{1}=0 & \text { on } \partial \Omega_{1} \\ w_{1}(0, x)=0 & \text { in } \Omega_{1}\end{cases}
$$

The parabolic regularizing effect (see Lemma 4.1 for $m=0$ ) gives for this system

$$
\varepsilon \int_{0}^{t^{*}}\left\|w_{1}\right\|_{H_{0}^{1}\left(\Omega_{1}\right)}^{2} d t \leq \frac{1}{\varepsilon} \int_{0}^{t^{*}}\left\|\left[f^{\prime}(N)-f^{\prime}(u)\right]\left(w_{1, x}-\chi_{1}^{\prime} v\right)-\varepsilon\left(\chi_{1}^{\prime \prime} v+2 \chi_{1}^{\prime} v_{x}\right)\right\|_{H^{-1}\left(\Omega_{1}\right)}^{2} d t
$$

Let us now estimate each of the terms on the right hand-side. Denoting by $C_{\Omega_{1}}=\frac{\left|\Omega_{1}\right|}{\pi}$ (the Poincaré's constant of $\Omega_{1}$ ), and defining the $H^{-1}$ norm on $\Omega_{1}$ as in Section 4.1, we have for the first term

$$
\begin{gathered}
\int_{0}^{t^{*}}\left\|\left[f^{\prime}(N)-f^{\prime}(u)\right] w_{1, x}(t, \cdot)\right\|_{H^{-1}\left(\Omega_{1}\right)}^{2} d t \leq \int_{0}^{t^{*}}\left\|\left[f^{\prime}(N)-f^{\prime}(u)\right]\right\|_{H^{1}\left(\Omega_{1}\right)}^{2}\left\|w_{1, x}(t, \cdot)\right\|_{H^{-1}\left(\Omega_{1}\right)}^{2} d t \\
\leq \int_{0}^{t^{*}}\left(C\left|\Omega_{1}\right|\left\|f^{\prime}\right\|_{L^{\infty}(P, N)}^{2}+\left\|u_{x} f^{\prime \prime}(u)\right\|_{L^{2}\left(\Omega_{1}\right)}^{2}\right)\left\|w_{1}(t, \cdot)\right\|_{L^{2}\left(\Omega_{1}\right)}^{2} d t \\
\leq \int_{0}^{t^{*}}\left(C\left|\Omega_{1}\right|\left\|f^{\prime}\right\|_{L^{\infty}(P, N)}^{2}+\left\|f^{\prime \prime}\right\|_{L^{\infty}(P, N)}^{2}\left\|u_{x}\left(t, \cdot+f^{\prime}(N) t\right)\right\|_{L^{2}\left(\Omega_{1}\right)}^{2}\right)\left|\Omega_{1}\right|\|v\|_{L^{\infty}\left(\Omega_{1}\right)}^{2} d t .
\end{gathered}
$$


It only remains to estimate $\left\|u_{x}\left(t, \cdot+f^{\prime}(N) t\right)\right\|_{L^{2}\left(\Omega_{1}\right)}^{2}$. For this, we consider another bounded open set $\tilde{\Omega}_{1}$ such that $\Omega_{1}=\left(a_{1}, b_{1}\right) \subset\left[a_{1}, b_{1}+f^{\prime}(N) t^{*}\right] \subset \tilde{\Omega}_{1}$. We take $\tilde{\chi}_{1} \in \mathcal{C}_{c}^{\infty}\left(\tilde{\Omega}_{1}\right)$ such that $\tilde{\chi}_{1}=1$ on $\left[a_{1}, b_{1}+f^{\prime}(N) t^{*}\right]$ and set $y_{1}(t, x)=\tilde{\chi}_{1}(x) u(t, x)$. Since $y_{1}$ satisfies

$$
y_{1, t}-\varepsilon y_{1, x x}=-\tilde{\chi}_{1}[f(u)]_{x}-\varepsilon\left(\tilde{\chi}_{1}^{\prime \prime} u+2 \tilde{\chi}_{1}^{\prime} u_{x}\right),
$$

the parabolic regularizing effect (see Lemma 4.1 for $m=0$ ) gives

$$
\varepsilon^{2} \int_{0}^{t^{*}}\left\|y_{1}\right\|_{H_{0}^{1}\left(\tilde{\Omega}_{1}\right)}^{2} d t \leq \varepsilon\left\|u_{0}\right\|_{L^{2}\left(\tilde{\Omega}_{1}\right)}^{2}+C \int_{0}^{t^{*}}\|f(u)\|_{L^{2}\left(\tilde{\Omega}_{1}\right)}^{2} d t+C\left|\tilde{\Omega}_{1}\right|^{2} \varepsilon^{2} \int_{0}^{t^{*}}\|u\|_{L^{2}\left(\tilde{\Omega}_{1}\right)}^{2} d t,
$$

and hence,

$$
\int_{0}^{t^{*}}\left\|u_{x}\left(t, \cdot+f^{\prime}(N) t\right)\right\|_{L^{2}\left(\Omega_{1}\right)}^{2} d t \leq \frac{\left|\tilde{\Omega}_{1}\right| t^{*}}{\varepsilon^{2}}\left(\|f\|_{L^{\infty}(P, N)}^{2}+\varepsilon \max \left(|P|^{2},|N|^{2}\right)\right) .
$$

Coming back to (19) and using estimate (17) on $v$, this yields

$$
\begin{aligned}
& \int_{0}^{t^{*}}\left\|\left[f^{\prime}(N)-f^{\prime}(u)\right] w_{1, x}(t, \cdot)\right\|_{H^{-1}\left(\Omega_{1}\right)}^{2} d t \leq C\left(\left|\Omega_{1}\right|\left\|f^{\prime}\right\|_{L^{\infty}(P, N)}^{2}+\frac{\left|\tilde{\Omega}_{1}\right|}{\varepsilon^{2}}\left\|f^{\prime \prime}\right\|_{L^{\infty}(P, N)}^{2}\|f\|_{L^{\infty}(P, N)}^{2}\right) \\
& \times\left|\Omega_{1}\right| t^{*}(N-P)^{2} e^{-\frac{2}{\varepsilon}\left(\sigma_{m}-s\right)\left(f^{\prime}(N)-s\right)(\mu-1) t^{*}}, \quad(20)
\end{aligned}
$$

Concerning the other terms in (18), we simply have

$$
\begin{aligned}
& \int_{0}^{t^{*}}\left\|\left[f^{\prime}(N)-f^{\prime}(u)\right] \chi_{1}^{\prime} v-\varepsilon\left(\chi_{1}^{\prime \prime} v+2 \chi_{1}^{\prime} v_{x}\right)\right\|_{H^{-1}\left(\Omega_{1}\right)}^{2} d t \\
& \leq C \int_{0}^{t^{*}}\left(\left\|f^{\prime}\right\|_{L^{\infty}(P, N)}^{2}\left|\Omega_{1}\right|^{2}+\left|\Omega_{1}\right|^{2} \varepsilon^{2}+\varepsilon^{2}\right)\|v\|_{L^{2}\left(\Omega_{1}\right)}^{2} d t \\
& \quad \leq C\left\|f^{\prime}\right\|_{L^{\infty}(P, N)}^{2}\left|\Omega_{1}\right|^{3} t^{*}(N-P)^{2} e^{-\frac{2}{\varepsilon}\left(\sigma_{m}-s\right)\left(f^{\prime}(N)-s\right)(\mu-1) t^{*}},
\end{aligned}
$$

after using estimate (17) on $v$.

Now, replacing (20) and (21) in (18), we obtain

$$
\begin{array}{r}
\int_{0}^{t^{*}}\left\|w_{1}\right\|_{H_{0}^{1}\left(\Omega_{1}\right)}^{2} d t \leq C \frac{\left|\tilde{\Omega}_{1}\right|^{3}}{\varepsilon^{4}}\left(\left\|f^{\prime}\right\|_{L^{\infty}(P, N)}^{2}+\left\|f^{\prime \prime}\right\|_{L^{\infty}(P, N)}^{2}\|f\|_{L^{\infty}(P, N)}^{2}\right) \\
\times t^{*}(N-P)^{2} e^{-\frac{2}{\varepsilon}\left(\sigma_{m}-s\right)\left(f^{\prime}(N)-s\right)(\mu-1) t^{*}} .
\end{array}
$$

We now take $\chi_{2} \in \mathcal{C}_{c}^{\infty}\left(\Omega_{2}\right)$ and set $w_{2}(t, x)=\chi_{2}(x) w_{1}(t, x)=\chi_{2}(x) v(t, x)$, that satisfies

$$
\begin{cases}w_{2, t}-\varepsilon w_{2, x x}=\left[f^{\prime}(N)-f^{\prime}(u)\right] \chi_{2} w_{1, x}-\varepsilon\left(\chi_{2}^{\prime \prime} w_{1}+2 \chi_{2}^{\prime} w_{1, x}\right) & \text { in }\left(0, t^{*}\right) \times \Omega_{1} \\ w_{2}=0 & \text { on } \partial \Omega_{1} \\ w_{2}(0, x)=0 & \text { in } \Omega_{1}\end{cases}
$$

The parabolic regularizing effect (see Lemma 4.1 for $m=1$ ) gives for this system

$$
\left\|w_{2}\left(t^{*}, \cdot\right)\right\|_{H_{0}^{1}\left(\Omega_{1}\right)}^{2} \leq \frac{1}{\varepsilon} \int_{0}^{t^{*}}\left\|\left[f^{\prime}(N)-f^{\prime}(u)\right] \chi_{2} w_{1, x}-\varepsilon\left(\chi_{2}^{\prime \prime} w_{1}+2 \chi_{2}^{\prime} w_{1, x}\right)\right\|_{L^{2}\left(\Omega_{1}\right)}^{2} d t,
$$

which directly yields

$$
\left\|w_{2}\left(t^{*}, \cdot\right)\right\|_{H_{0}^{1}\left(\Omega_{1}\right)}^{2} \leq \frac{C}{\varepsilon}\left\|f^{\prime}\right\|_{L^{\infty}(P, N)}^{2} \int_{0}^{t^{*}}\left\|w_{1}\right\|_{H_{0}^{1}\left(\Omega_{1}\right)}^{2} d t .
$$


As a consequence of (22), we thus have, for $t^{*}>0$,

$$
\begin{aligned}
\left\|w_{2}\left(t^{*}, \cdot\right)\right\|_{H^{1}\left(\Omega_{2}\right)} \leq & C \frac{\left|\tilde{\Omega}_{1}\right|^{\frac{3}{2}}}{\varepsilon^{\frac{5}{2}}} \sqrt{t^{*}}(N-P)\left\|f^{\prime}\right\|_{L^{\infty}(P, N)} \\
& \times\left(\left\|f^{\prime}\right\|_{L^{\infty}(P, N)}+\left\|f^{\prime \prime}\right\|_{L^{\infty}(P, N)}\|f\|_{L^{\infty}(P, N)}\right) e^{-\frac{1}{\varepsilon}\left(\sigma_{m}-s\right)\left(f^{\prime}(N)-s\right)(\mu-1) t^{*}} .
\end{aligned}
$$

Note that we could have proved the same type of estimate for the $H^{2}$ norm, but not more since we only supposed $f \in W_{\text {loc }}^{2, \infty}$. However, if $f$ is more regular, we can prove the estimate in higher regularity spaces.

Now, to come back to $u$, we choose the sets $\Omega_{1}, \Omega_{2}$ and the function $\chi_{2}$ such that $\chi_{2}=1$ on $\left(-f^{\prime}(N) t^{*}, \beta^{*}-\kappa\right) \subset \Omega_{2} \subset \Omega_{1}=\left(-f^{\prime}(N) t^{*}-\kappa, \beta^{*}\right)$ for some constant $\kappa>0$, and for $t^{*}>0$ satisfying $\beta^{*}-\kappa>-f^{\prime}(N) t^{*}$ (i.e., $\left.\left[\mu s+(1-\mu) f^{\prime}(N)\right] t^{*}>\kappa\right)$. Note that $\left|\Omega_{1}\right|=\beta^{*}+\kappa+f^{\prime}(N) t^{*}=$ $\kappa+\left[\mu s+(1-\mu) f^{\prime}(N)\right] t^{*}$ depends on $t^{*}$ and on $N$. Estimate (23) now yields for all $\kappa>0$ and $t^{*}>\frac{\kappa}{\mu s+(1-\mu) f^{\prime}(N)}$,

$$
\left\|v\left(t^{*}, \cdot\right)\right\|_{H^{1}\left(-f^{\prime}(N) t^{*}, \beta^{*}-\kappa\right)} \leq D e^{-\frac{1}{\varepsilon}\left(\sigma_{m}-s\right)\left(f^{\prime}(N)-s\right)(\mu-1) t^{*}},
$$

with

$$
D=C_{\kappa} \frac{t^{* 2}}{\varepsilon^{\frac{5}{2}}}(N-P)\left\|f^{\prime}\right\|_{L^{\infty}(P, N)}^{\frac{5}{2}}\left(\left\|f^{\prime}\right\|_{L^{\infty}(P, N)}+\left\|f^{\prime \prime}\right\|_{L^{\infty}(P, N)}\|f\|_{L^{\infty}(P, N)}\right) .
$$

Recalling the expression of $\beta^{*}$ and $v$, we obtain

$$
\begin{aligned}
\left\|u\left(t^{*}, \cdot\right)-N\right\|_{H^{1}\left(0,-\mu\left(f^{\prime}(N)-s\right) t^{*}+f^{\prime}(N) t^{*}-\kappa\right)} & \leq D e^{-\frac{1}{\varepsilon}\left(\sigma_{m}-s\right)\left(f^{\prime}(N)-s\right)(\mu-1) t^{*}} \\
\left\|u\left(t^{*}, \cdot\right)-N\right\|_{H^{1}\left(0,\left[(1-\mu) f^{\prime}(N)+\mu s\right] t^{*}-\kappa\right)} & \leq D e^{-\frac{1}{\varepsilon}\left(\sigma_{m}-s\right)\left(f^{\prime}(N)-s\right)(\mu-1) t^{*}} .
\end{aligned}
$$

We now choose $\mu=1+\nu \frac{s}{f^{\prime}(N)-s}$ with $\nu \in(0,1)$, so that

$$
\left\{\begin{array}{l}
(\mu-1)=\nu \frac{s}{f^{\prime}(N)-s}>0 \\
(1-\mu) f^{\prime}(N)+\mu s=(1-\nu) s>0 .
\end{array}\right.
$$

Replacing this in $(24)$ gives for any $\kappa>0, \nu \in(0,1)$ and $t^{*}>\frac{\kappa}{(1-\nu) s}$,

$$
\left\|u\left(t^{*}, \cdot\right)-N\right\|_{H^{1}\left(0,(1-\nu) s t^{*}-\kappa\right)} \leq D e^{-\frac{\nu}{\varepsilon} s\left(\sigma_{m}-s\right) t^{*}} .
$$

Finally, on $(0, L)$, we obtain, for any $\kappa>0, \nu \in(0,1)$ and $t^{*}>\frac{L+\kappa}{(1-\nu) s}$,

$$
\left\|u\left(t^{*}, \cdot\right)-N\right\|_{H^{1}(0, L)} \leq D e^{-\frac{\nu}{\varepsilon} s\left(\sigma_{m}-s\right) t^{*}},
$$

and the proposition is proved.

\subsection{Approximate controllability using a rarefaction wave}

We here prove the approximate controllability from a large state $N$ to the state $M$, thanks to a rarefaction wave.

Proposition 2.4. Suppose that $f^{\prime \prime} \geq 0$ on the interval $(M, N)$ and $f^{\prime}(M)>0$. Then, for all $\varepsilon_{0}>0, \kappa>0$, and $t^{*}>\frac{L+\kappa}{f^{\prime}(M)}$, there exists a constant $\delta\left(t^{*}\right)=\delta\left(t^{*}, \kappa, \varepsilon_{0}, M, N\right)>0$ such that for all $\varepsilon \in\left(0, \varepsilon_{0}\right)$, there exist control functions $g_{0}$ and $g_{L}$ with

$$
\left\|g_{0}\right\|_{L^{\infty}\left(0, t^{*}\right)} \leq \max (|M|,|N|) \quad \text { and } \quad\left\|g_{L}\right\|_{L^{\infty}\left(0, t^{*}\right)} \leq \max (|M|,|N|),
$$

such that the solution $u$ of (1), (2) with initial condition $\left.u\right|_{t=0}=N$ satisfies,

$$
\left\|u\left(t^{*}, \cdot\right)-M\right\|_{H^{1}(0, L)} \leq \frac{\delta\left(t^{*}\right)}{\varepsilon^{\frac{3}{2}}} \exp \left(-\frac{1}{4 \varepsilon t^{*}}\left(f^{\prime}(M) t^{*}-L-\kappa\right)^{2}\right) .
$$


As for the proof of Proposition 2.4, the solution $u$ is obtained by taking the restriction to $\left(0, t^{*}\right) \times$ $(0, L)$ of the solution defined on whole $\mathbb{R}_{+} \times \mathbb{R}$ (still denoted $u$ ) of the following problem (see Figure 2)

$$
\begin{cases}u_{t}+[f(u)]_{x}-\varepsilon u_{x x}=0 & \text { in } \mathbb{R}_{+} \times \mathbb{R} \\ \left.u\right|_{t=0}=u_{0} & \text { in } \mathbb{R}\end{cases}
$$

with

$$
u_{0}(x)\left\{\begin{array}{l}
=M \quad \text { if } x \leq-k \\
=N \quad \text { if } x \geq 0 \\
\in \mathcal{C}^{\infty}([-k, 0]), \text { increasing }
\end{array}\right.
$$

for some (small) $k>0$.

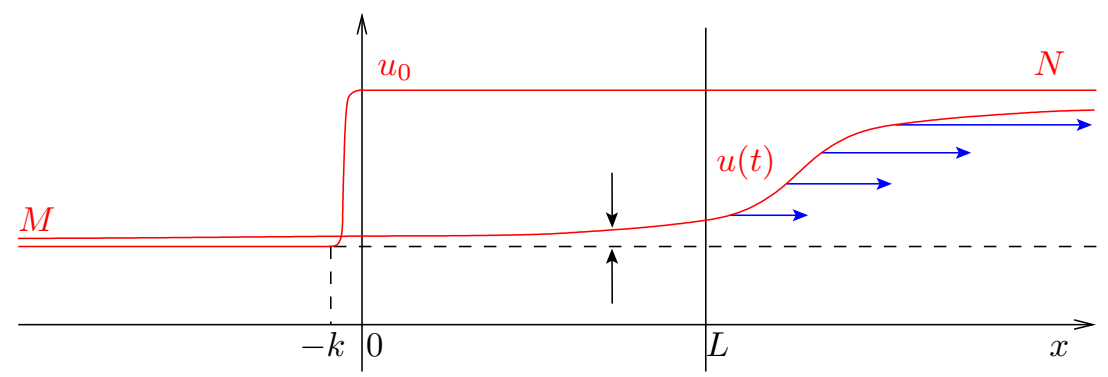

Figure 2: Rarefaction wave.

Initial data, and solution after a time $t>\frac{L}{f^{\prime}(M)}$ has gone by.

Then, the control functions $g_{0}$ and $g_{L}$ are obtained by taking the traces of $u$ along the lines $\left(0, t^{*}\right) \times\{0\}$ and $\left(0, t^{*}\right) \times\{L\}$. As in Section 2.1, (25) follows directly from the comparison principle $[10$, Theorem 6.3 .2$]$, and we hence only have to prove (26).

To that purpose, we first prove the following lemma, that gives a $L^{2}$ estimate of the convergence rate of a viscous rarefaction wave to the associated non-viscous one. Its proof is inspired by [11, Theorem 1.1.], where the author proves dissipation results for the Navier-Stokes equations and associated vortex patches in the vanishing viscosity limit.

Lemma 2.5. Let $u$ be the solution of the problem (27), (28). Then for all $\varepsilon_{0}>0, k>0$, and $t^{*} \geq 0$, there exists $\gamma\left(t^{*}\right)=\gamma\left(t^{*}, k, \varepsilon_{0}, M, N\right)>0$ (nondecreasing with respect to $t^{*}$ ) such that for any $\varepsilon \in\left(0, \varepsilon_{0}\right)$ and $\eta>0$,

$$
\left\|u\left(t^{*}, \cdot\right)-M\right\|_{L^{2}\left(-\infty,-\eta-k+f^{\prime}(M) t^{*}\right)}+\left\|u\left(t^{*}, \cdot\right)-N\right\|_{L^{2}\left(\eta+f^{\prime}(N) t^{*},+\infty\right)} \leq \gamma\left(t^{*}\right) e^{-\frac{\eta^{2}}{4 \varepsilon t^{*}}} .
$$

Note that the function $\gamma\left(t^{*}\right)$ is relatively explicit, i.e.,

$$
\gamma\left(t^{*}\right)=\|X\|_{L^{2}\left(0, t^{*} ; L^{2}(\mathbb{R})\right)} e^{\frac{t^{*}}{2}\left(\left\|f^{\prime \prime}\right\|_{L^{\infty}(M, N)}\left\|u_{0, x}\right\|_{L^{\infty}(\mathbb{R})}+1\right)},
$$

where the function $X$ can be estimated uniformly with respect to $\varepsilon$, as in (31).

Proof. We first consider a function $w \in \mathcal{C}^{\infty}\left(\mathbb{R}_{+} \times \mathbb{R}\right)$ satisfying

$$
\begin{cases}w(t, x)=M & \text { if } x \leq-k+f^{\prime}(M) t, \quad t \geq 0 \\ w(t, x)=N & \text { if } x \geq f^{\prime}(N) t, \quad t \geq 0 \\ w(t, \cdot) & \text { is increasing on }\left[-k+f^{\prime}(M) t, f^{\prime}(N) t\right], \quad t \geq 0 \\ \left.w\right|_{t=0}=u_{0} & \text { in } \mathbb{R} .\end{cases}
$$

We shall make estimates on $v:=u-w$, that satisfies the problem

$$
\begin{cases}v_{t}+f^{\prime}(u) v_{x}-\varepsilon v_{x x}=-w_{t}-f^{\prime}(u) w_{x}+\varepsilon w_{x x} & \text { in } \mathbb{R}_{+} \times \mathbb{R} \\ \left.v\right|_{t=0}=0 & \text { in } \mathbb{R}\end{cases}
$$


We set

$$
X(t, x):=-w_{t}-f^{\prime}(u) w_{x}+\varepsilon w_{x x},
$$

and notice that for all $t \geq 0, X$ has a compact support with respect to $x$, included in $[-k+$ $\left.f^{\prime}(M) t, f^{\prime}(N) t\right]$. Hence, for $t \geq 0, X(t, \cdot) \in L^{2}(\mathbb{R})$ and we have the estimate

$$
\|X(t, \cdot)\|_{L^{2}} \leq\left\|w_{t}(t, \cdot)\right\|_{L^{2}}+f^{\prime}(N)\left\|w_{x}(t, \cdot)\right\|_{L^{2}}+\varepsilon_{0}\left\|w_{x x}(t, \cdot)\right\|_{L^{2}}
$$

Moreover, the right hand-side of (31) is continuous with respect to $t$, so that $X \in L_{\text {loc }}^{2}\left(\mathbb{R}_{+} ; L^{2}(\mathbb{R})\right)$, uniformly with $\varepsilon$.

We define $\psi$ the flow associated with the vector field $f^{\prime}(u)$, i.e., the solution of

$$
\psi(t, x)=x+\int_{0}^{t} f^{\prime}(u)(s, \psi(s, x)) d s,
$$

and $\psi^{-1}$ the associated backward flow. Following [11], we set, for $\alpha \in \mathbb{R}$,

$$
\Phi(t, x)=\exp \left(\alpha g\left(\psi^{-1}(t, x)\right)\right),
$$

so that the function $\Phi$ is constant along flow lines, that is $\frac{d}{d t}(\Phi(t, \psi(t, x)))=0$. Here, the function $g \in W_{\mathrm{loc}}^{1, \infty}(\mathbb{R})$ and the constant $\alpha=\alpha\left(t^{*}\right)$ will be chosen later on. We suppose moreover that $g$ is equal to a constant $R$ outside a compact set (see the definition in (36)). In particular, this yields $\Phi \in \mathcal{C}^{0}\left(\mathbb{R}_{+} ; W^{1, \infty}(\mathbb{R})\right)$ and $\Phi v \in \mathcal{C}^{0}\left(\mathbb{R}_{+} ; H^{1}(\mathbb{R})\right)$ since $v \in \mathcal{C}^{0}\left(\mathbb{R}_{+} ; H^{1}(\mathbb{R})\right)$ from (30). As a consequence of (30), $\Phi v$ satisfies the following equation

$$
(\Phi v)_{t}+f^{\prime}(u)(\Phi v)_{x}-\varepsilon(\Phi v)_{x x}=v\left(\Phi_{t}+f^{\prime}(u) \Phi_{x}\right)-\varepsilon v \Phi_{x x}-2 \varepsilon v_{x} \Phi_{x}+X .
$$

Since $\Phi$ is constant along flow lines, the first term on the right hand-side vanishes. Next, taking the inner product of this equation with $\Phi v$ yields

$$
\frac{1}{2} \frac{d}{d t}\|\Phi v\|_{L^{2}}^{2}+\int_{\mathbb{R}} f^{\prime}(u)(\Phi v)_{x} \Phi v+\varepsilon\left\|(\Phi v)_{x}\right\|_{L^{2}}^{2}=-\varepsilon \int_{\mathbb{R}} v^{2} \Phi_{x x} \Phi-2 \varepsilon \int_{\mathbb{R}} v_{x} \Phi_{x} \Phi v+\int_{\mathbb{R}} X \Phi v .
$$

In this expression, we have, after integrations by parts,

$$
\int_{\mathbb{R}} f^{\prime}(u)(\Phi v)_{x} \Phi v=-\frac{1}{2} \int_{\mathbb{R}}\left(f^{\prime}(u)\right)_{x}(\Phi v)^{2},
$$

and

$$
-\varepsilon \int_{\mathbb{R}} v^{2} \Phi_{x x} \Phi-2 \varepsilon \int_{\mathbb{R}} v_{x} \Phi_{x} \Phi v=2 \varepsilon \int_{\mathbb{R}} v_{x} \Phi_{x} \Phi v+\varepsilon \int_{\mathbb{R}}\left(v \Phi_{x}\right)^{2}-2 \varepsilon \int_{\mathbb{R}} v_{x} \Phi_{x} \Phi v=\varepsilon \int_{\mathbb{R}}\left(v \Phi_{x}\right)^{2} .
$$

Now, Equation (34) yields

$$
\frac{1}{2} \frac{d}{d t}\|\Phi v\|_{L^{2}}^{2} \leq \frac{1}{2} \int_{\mathbb{R}}\left(f^{\prime}(u)\right)_{x}(\Phi v)^{2}+\varepsilon\left\|v \Phi_{x}\right\|_{L^{2}}^{2}+\int_{\mathbb{R}} X \Phi v .
$$

We choose the function $g$ in (33) as

$$
g(x)=\min \{R, d(x,[-k, 0])\} \in W^{1, \infty}(\mathbb{R})
$$

for some constant $R>0$. Notice that we have $\left\|g^{\prime}\right\|_{L^{\infty}(\mathbb{R})}=1$. Then, $\Phi_{x}=\alpha\left(\psi^{-1}\right)_{x} g^{\prime} \circ \psi^{-1} \Phi$ can be estimated thanks to the following lemma.

Lemma 2.6. Let $u$ be the solution of (27), (28) and $\psi^{-1}$ the backward flow associated with the vector field $f^{\prime}(u)$. Then we have, for any $t \geq 0$ and $\varepsilon>0$,

$$
0 \leq u_{x}(t, x) \leq\left\|u_{0, x}\right\|_{L^{\infty}(\mathbb{R})}
$$

and

$$
\left\|\left(\psi^{-1}\right)_{x}(t, \cdot)\right\|_{L^{\infty}(\mathbb{R})} \leq 1 .
$$


Note that the estimates (37) and (38) are consequences of the fact that the solution of (27), (28) is a rarefaction wave. The proof of this lemma is postponed to the end of this proof.

As a consequence of Lemma 2.6, we now have

$$
\left\|\left(f^{\prime}(u)\right)_{x}\right\|_{L^{\infty}(\mathbb{R})} \leq\left\|f^{\prime \prime}\right\|_{L^{\infty}(M, N)}\left\|u_{0, x}\right\|_{L^{\infty}(\mathbb{R})},
$$

from (37) and

$$
\left\|\alpha\left(\psi^{-1}\right)_{x} g^{\prime} \circ \psi^{-1}\right\|_{L^{\infty}\left(\mathbb{R}_{+} \times \mathbb{R}\right)} \leq \alpha\left\|\left(\psi^{-1}\right)_{x}\right\|_{L^{\infty}}\left\|g^{\prime}\right\|_{L^{\infty}} \leq \alpha,
$$

from (38). Coming back to (35), estimates (39) and (40) yield

$$
\frac{1}{2} \frac{d}{d t}\|\Phi v\|_{L_{x}^{2}}^{2} \leq \frac{\left\|f^{\prime \prime}\right\|_{L^{\infty}(M, N)}\left\|u_{0, x}\right\|_{L^{\infty}(\mathbb{R})}}{2}\|\Phi v\|_{L_{x}^{2}}^{2}+\varepsilon \alpha^{2}\|\Phi v\|_{L_{x}^{2}}^{2}+\frac{1}{2}\|X\|_{L_{x}^{2}}^{2}+\frac{1}{2}\|\Phi v\|_{L_{x}^{2}}^{2} .
$$

Now, using Gronwall's lemma in

$$
\frac{d}{d t}\|\Phi v\|_{L_{x}^{2}}^{2} \leq\left(2 \varepsilon \alpha^{2}+\left\|f^{\prime \prime}\right\|_{L^{\infty}(M, N)}\left\|u_{0, x}\right\|_{L^{\infty}(\mathbb{R})}+1\right)\|\Phi v\|_{L_{x}^{2}}^{2}+\|X\|_{L_{x}^{2}}^{2}
$$

yields, for any $t^{*}>0$,

$$
\left\|\Phi v\left(t^{*}, \cdot\right)\right\|_{L^{2}}^{2} \leq\|X\|_{L^{2}\left(0, t^{*} ; L^{2}(\mathbb{R})\right)}^{2} \exp \left[\left(2 \varepsilon \alpha^{2}+\left\|f^{\prime \prime}\right\|_{L^{\infty}(M, N)}\left\|u_{0, x}\right\|_{L^{\infty}(\mathbb{R})}+1\right) t^{*}\right],
$$

since $\left.(\Phi v)\right|_{t=0}=0$. We set $\gamma\left(t^{*}\right)^{2}=\|X\|_{L^{2}\left(0, t^{*} ; L^{2}(\mathbb{R})\right)}^{2} e^{\left(\left\|f^{\prime \prime}\right\|_{L^{\infty}(M, N)}\left\|u_{0, x}\right\|_{L^{\infty}(\mathbb{R})}+1\right) t^{*}}$ which does not depend on $\varepsilon, \alpha$. It depends only on $\varepsilon_{0}$ and $k$ through the initial condition $u_{0}$ and $X$.

Let us now take $\eta \in(0, R)$. If $x \in \psi\left(t^{*},(-\infty,-k-\eta) \cup(\eta,+\infty)\right)$, then $\psi^{-1}\left(t^{*}, x\right) \in(-\infty,-k-$ $\eta) \cup(\eta,+\infty)$ and $g \circ \psi^{-1}\left(t^{*}, x\right) \geq \eta$. Thus, $\Phi\left(t^{*}, x\right)=e^{\alpha g \circ \psi^{-1}\left(t^{*}, x\right)} \geq e^{\alpha \eta}$ so that

$$
\left\|\Phi v\left(t^{*}, \cdot\right)\right\|_{L^{2}(\mathbb{R})} \geq e^{\alpha \eta}\left\|v\left(t^{*}, \cdot\right)\right\|_{L^{2}\left(\psi\left(t^{*},(-\infty,-k-\eta) \cup(\eta,+\infty)\right)\right)} .
$$

This, together with (42) gives

$$
\begin{gathered}
\left\|v\left(t^{*}, \cdot\right)\right\|_{L^{2}\left(\psi\left(t^{*},(-\infty,-k-\eta) \cup(\eta,+\infty)\right)\right)} \leq \gamma\left(t^{*}\right) e^{\varepsilon \alpha^{2} t^{*}-\alpha \eta}, \\
\left\|v\left(t^{*}, \cdot\right)\right\|_{L^{2}\left(\left(-\infty, \psi\left(t^{*},-k-\eta\right)\right) \cup\left(\psi\left(t^{*}, \eta\right),+\infty\right)\right)} \leq \gamma\left(t^{*}\right) e^{-\frac{\eta^{2}}{4 \varepsilon t^{*}},}
\end{gathered}
$$

after having chosen $\alpha=\frac{\eta}{2 \varepsilon t^{*}}$. This inequality does not depend on $R$, so making $R \rightarrow+\infty$, we see that (43) holds for any $\eta>0$.

It only remains to prove that $\left(-\infty,-k-\eta+f^{\prime}(M) t^{*}\right) \cup\left(\eta+f^{\prime}(N) t^{*},+\infty\right) \subset\left(-\infty, \psi\left(t^{*},-k-\right.\right.$ $\left.\eta)) \cup\left(\psi\left(t^{*}, \eta\right),+\infty\right)\right)$. Actually, this is a direct consequence of

$$
\begin{gathered}
\psi\left(t^{*}, \eta\right)=\eta+\int_{0}^{t^{*}} f^{\prime}(u)(t, \psi(t, \eta)) d t \leq \eta+f^{\prime}(N) t^{*}, \\
\psi\left(t^{*},-k-\eta\right)=-k-\eta+\int_{0}^{t^{*}} f^{\prime}(u)(t, \psi(t,-k-\eta)) d t \geq-k-\eta+f^{\prime}(M) t^{*},
\end{gathered}
$$

where we have used the convexity of $f$ and the comparison priciple [10, Theorem 6.3.2] for the solutions of viscous conservation laws. This concludes the proof of Lemma 2.5.

We now have to prove Lemma 2.6.

Proof of Lemma 2.6. Firstly, we check that for any $\varepsilon>0$ and $t \geq 0$, the speed $f^{\prime}(u)$ is nondecreasing, i.e., $\left(f^{\prime}(u)\right)_{x} \geq 0$. Since $f^{\prime \prime} \geq 0$, we only have to prove that $u_{x}$ is nonnegative. The function $y=u_{x}$ is solution of

$$
\begin{cases}y_{t}-\varepsilon y_{x x}+f^{\prime}(u) y_{x}+f^{\prime \prime}(u) y^{2}=0 & \text { in } \mathbb{R}_{+} \times \mathbb{R} \\ \left.y\right|_{t=0}=\left.u_{x}\right|_{t=0} \geq 0 & \text { in } \mathbb{R}\end{cases}
$$

As a consequence of the weak maximum principle for parabolic equations (see for instance $[12, \mathrm{p}$. $368])$, we have $u_{x}(t, x)=y(t, x) \geq 0$ in $\mathbb{R}_{+} \times \mathbb{R}$, and

$$
\left(f^{\prime}(u)\right)_{x}(t, x) \geq 0, \quad(t, x) \in \mathbb{R}_{+} \times \mathbb{R} .
$$


Secondly, since $f^{\prime \prime} \geq 0$, Equation (44) yields

$$
\left\{\begin{array}{l}
y_{t}-\varepsilon y_{x x}+f^{\prime}(u) y_{x} \leq 0 \text { in } \mathbb{R}_{+} \times \mathbb{R} \\
\left.y\right|_{t=0}=\left.u_{x}\right|_{t=0} \in L^{\infty}(\mathbb{R}) .
\end{array}\right.
$$

For this system, the same maximum principle gives $u_{x}(t, x)=y(t, x) \leq\left\|\left.y\right|_{t=0}\right\|_{L^{\infty}(\mathbb{R})}=\left\|\left.u_{x}\right|_{t=0}\right\|_{L^{\infty}(\mathbb{R})}$. Hence, we have

$$
0 \leq u_{x}(t, x) \leq\left\|\left.u_{x}\right|_{t=0}\right\|_{L^{\infty}(\mathbb{R})}, \quad(t, x) \in \mathbb{R}_{+} \times \mathbb{R},
$$

and (37) is proved.

Finally, to prove (38), we recall that the backward flow $\psi^{-1}$ is given by

$$
\psi^{-1}(t, x)=x-\int_{0}^{t} f^{\prime}(u)\left(s, \psi^{-1}(s, x)\right) d s, \quad(t, x) \in \mathbb{R}_{+} \times \mathbb{R},
$$

which yields, derivating with respect to $x$ and $t$,

$$
\left\{\begin{array}{l}
\frac{d}{d t}\left(\psi^{-1}\right)_{x}(t, x)=-\left(\psi^{-1}\right)_{x}(t, x) \cdot\left(f^{\prime}(u)\right)_{x}\left(t, \psi^{-1}(t, x)\right), \quad(t, x) \in \mathbb{R}_{+} \times \mathbb{R}, \\
\left(\psi^{-1}\right)_{x}(0, x)=1 .
\end{array}\right.
$$

This can be rewritten under the form $\left(\psi^{-1}\right)_{x}(t, x)=\exp \left(-\int_{0}^{t}\left(f^{\prime}(u)\right)_{x}\left(s, \psi^{-1}(s, x)\right) d s\right)$, which, thanks to (45), yields

$$
0 \leq\left(\psi^{-1}\right)_{x}(t, x) \leq 1, \quad(t, x) \in \mathbb{R}_{+} \times \mathbb{R},
$$

and Lemma 2.6 is proved.

As a consequence of Lemma 2.5 and using a bootstrap argument, we are now able to prove the central result of this section.

Proof of Proposition 2.4. We study the evolution of $(27)$, (28) for $t \in\left(0, t^{*}\right)$. First, we set

$$
v(t, x)=(u-M)\left(t, x+f^{\prime}(M) t\right),
$$

for which estimate (29) of Lemma 2.5 yields, for any $\eta>0$,

$$
\|v(t, \cdot)\|_{L^{2}(-\infty,-k-\eta)} \leq \gamma(t) e^{-\frac{\eta^{2}}{4 \varepsilon t}}, \quad t \geq 0 .
$$

As in the proof of Proposition 2.1, we denote by $\Omega_{1}$ a bounded open interval of $(-\infty,-k-\eta)$ and $\chi_{1} \in \mathcal{C}_{c}^{\infty}\left(\Omega_{1}\right)$ a cut-off function satisfying $\chi_{1}=1$ on $\Omega_{2}$ with $\bar{\Omega}_{2} \subset \Omega_{1}$. The function $w_{1}(t, x)=$ $\chi_{1}(x) v(t, x)$ satisfies

$$
\begin{cases}w_{1, t}-\varepsilon w_{1, x x}=\left[f^{\prime}(M)-f^{\prime}(u)\right] \chi_{1} v_{x}-\varepsilon\left(\chi_{1}^{\prime \prime} v+2 \chi_{1}^{\prime} v_{x}\right) & \text { in }\left(0, t^{*}\right) \times \Omega_{1} \\ w_{1}=0 & \text { on } \partial \Omega_{1} \\ w_{1}(0, x)=0 & \text { in } \Omega_{1}\end{cases}
$$

The parabolic regularizing effect (see Lemma 4.1 for $m=0$ ) gives for this system

$$
\varepsilon \int_{0}^{t^{*}}\left\|w_{1}\right\|_{H_{0}^{1}\left(\Omega_{1}\right)}^{2} d t \leq \frac{1}{\varepsilon} \int_{0}^{t^{*}}\left\|\left[f^{\prime}(M)-f^{\prime}(u)\right] \chi_{1} v_{x}-\varepsilon\left(\chi_{1}^{\prime \prime} v+2 \chi_{1}^{\prime} v_{x}\right)\right\|_{H^{-1}\left(\Omega_{1}\right)}^{2} d t .
$$

Let us now estimate each of the terms on the right hand-side as in the proof of Proposition 2.1. Here however, we see that thanks to Lemma 2.6, it is not necessary to perform a preliminary $H^{1}$ estimate on $u$, as opposed to the proof of Proposition 2.1. The first term is

$$
\left\|\left[f^{\prime}(M)-f^{\prime}(u)\right] \chi_{1} v_{x}\right\|_{H^{-1}\left(\Omega_{1}\right)} \leq C\left(\left\|f^{\prime}\right\|_{L^{\infty}(M, N)}+\left\|\left(f^{\prime}(u)\right)_{x}\right\|_{L^{\infty}\left(\Omega_{1}\right)}\right)\|v\|_{L^{2}\left(\Omega_{1}\right)} .
$$

As a consequence of Lemma 2.6, we have $\left\|\left(f^{\prime}(u)\right)_{x}\right\|_{L^{\infty}(\mathbb{R})} \leq\left\|f^{\prime \prime}\right\|_{L^{\infty}(M, N)}\left\|u_{0, x}\right\|_{L^{\infty}(\mathbb{R})}$, so that

$$
\left\|\left[f^{\prime}(M)-f^{\prime}(u)\right] \chi_{1} v_{x}\right\|_{H^{-1}\left(\Omega_{1}\right)} \leq C\|v\|_{L^{2}\left(\Omega_{1}\right)} .
$$


Concerning the other terms, we have

$$
\varepsilon\left\|\left(\chi_{1}^{\prime \prime} v+2 \chi_{1}^{\prime} v_{x}\right)\right\|_{H^{-1}\left(\Omega_{1}\right)} \leq \varepsilon C\left(C_{\Omega_{1}}+1\right)\|v\|_{L^{2}\left(\Omega_{1}\right)},
$$

where the constants denoted by $C$ do not depend on $\Omega_{1}$ and $C_{\Omega_{1}}=\frac{\left|\Omega_{1}\right|}{\pi}$ as before. Coming back to (47) and using (46), we now have

$$
\int_{0}^{t^{*}}\left\|w_{1}\right\|_{H_{0}^{1}\left(\Omega_{1}\right)}^{2} d t \leq C\left(\frac{1}{\varepsilon^{2}}+\left|\Omega_{1}\right|^{2}\right) \int_{0}^{t^{*}}\|v\|_{L^{2}\left(\Omega_{1}\right)}^{2} d t \leq C\left(\frac{1}{\varepsilon^{2}}+\left|\Omega_{1}\right|^{2}\right) t^{*} \gamma\left(t^{*}\right)^{2} e^{-\frac{\eta^{2}}{2 \varepsilon t^{*}}}
$$

from (46), since $\Omega_{1} \subset(-\infty,-k-\eta)$ the functions $\gamma(t)$ and $e^{-\frac{\eta^{2}}{2 \varepsilon t}}$ are non-decreasing with respect to $t$.

We now take $\chi_{2} \in \mathcal{C}_{c}^{\infty}\left(\Omega_{2}\right)$ and set $w_{2}(t, x)=\chi_{2}(x) w_{1}(t, x)=\chi_{2}(x) v(t, x)$, that satisfies

$$
\begin{cases}w_{2, t}-\varepsilon w_{2, x x}=\left[f^{\prime}(M)-f^{\prime}(u)\right] \chi_{2} w_{1, x}-\varepsilon\left(\chi_{2}^{\prime \prime} w_{1}+2 \chi_{2}^{\prime} w_{1, x}\right) & \text { in }\left(0, t^{*}\right) \times \Omega_{1} \\ w_{2}=0 & \text { on } \partial \Omega_{1} \\ w_{2}(0, x)=0 & \text { in } \Omega_{1}\end{cases}
$$

The parabolic regularizing effect (see Lemma 4.1 for $m=1$ ) directly yields

$$
\left\|w_{2}\left(t^{*}, \cdot\right)\right\|_{H_{0}^{1}\left(\Omega_{1}\right)}^{2} \leq \frac{C}{\varepsilon} \int_{0}^{t^{*}}\left\|w_{1, x}\right\|_{L^{2}\left(\Omega_{1}\right)}^{2}+\varepsilon^{2}\left\|w_{1}\right\|_{L^{2}\left(\Omega_{1}\right)}^{2} d t \leq \frac{C}{\varepsilon} \int_{0}^{t^{*}}\left\|w_{1}\right\|_{H_{0}^{1}\left(\Omega_{1}\right)}^{2} d t .
$$

As a consequence of (48), we thus have

$$
\left\|w_{2}\left(t^{*}, \cdot\right)\right\|_{H_{0}^{1}\left(\Omega_{1}\right)}^{2} \leq \frac{C}{\varepsilon}\left(\frac{1}{\varepsilon^{2}}+\left|\Omega_{1}\right|^{2}\right) t^{*} \gamma\left(t^{*}\right)^{2} e^{-\frac{\eta^{2}}{2 \varepsilon t^{*}}} .
$$

Now, to come back to $u$, we choose the sets $\Omega_{1}, \Omega_{2}$, and the function $\chi_{2}$ such that $\chi_{2}=1$ on $\left(-f^{\prime}(M) t^{*},-\eta-2 k\right) \subset \Omega_{2} \subset \Omega_{1}=\left(-f^{\prime}(M) t^{*}-k,-\eta-k\right) \subset(-\infty,-\eta-k)$. With this choice, we have $\left|\Omega_{1}\right|=f^{\prime}(M) t^{*}-\eta$ and estimate (49) yields, for any $t^{*}>0$ and $0<\eta<f^{\prime}(M) t^{*}$,

$$
\left\|u\left(t^{*}, \cdot\right)-M\right\|_{H^{1}\left(0,-\eta-2 k+f^{\prime}(M) t^{*}\right)} \leq \frac{C}{\sqrt{\varepsilon}}\left(\frac{1}{\varepsilon}+\left|f^{\prime}(M) t^{*}-\eta\right|\right) \sqrt{t^{*}} \gamma\left(t^{*}\right) e^{-\frac{\eta^{2}}{4 \varepsilon t^{*}}} .
$$

It remains to choose $\eta$ so that $-\eta-2 k+f^{\prime}(M) t^{*}=L$, that is, $\eta=f^{\prime}(M) t^{*}-L-2 k$, which is positive as soon as $t^{*}>\frac{L+2 k}{f^{\prime}(M)}$. Finally, we have for any $t^{*}>\frac{L+2 k}{f^{\prime}(M)}$,

$$
\left\|u\left(t^{*}, \cdot\right)-M\right\|_{H^{1}(0, L)} \leq \frac{C}{\sqrt{\varepsilon}}\left(\frac{1}{\varepsilon}+(L+2 k)\right) \sqrt{t^{*}} \gamma\left(t^{*}\right) \exp \left(-\frac{1}{4 \varepsilon t^{*}}\left(f^{\prime}(M) t^{*}-L-2 k\right)^{2}\right),
$$

and Proposition 2.4 is proved, setting $\kappa=2 k$ and $\delta\left(t^{*}\right)=C \sqrt{t^{*}} \gamma\left(t^{*}\right)$.

Remark 2.7. This proposition and its proof need slight modifications when proving Proposition 1.7, since the right hand-side of (26) needs to be exponentially decreasing as $t^{*} \rightarrow+\infty$. For this, we first replace $\frac{1}{2}\|X\|_{L_{x}^{2}}^{2}+\frac{1}{2}\|\Phi v\|_{L_{x}^{2}}^{2}$ in Estimate (41) by $\frac{1}{2 \mu}\|X\|_{L_{x}^{2}}^{2}+\frac{\mu}{2}\|\Phi v\|_{L_{x}^{2}}^{2}$ for all $\mu>0$. Choosing $u_{0}$ such that $\left\|u_{0, x}\right\|_{L^{\infty}(\mathbb{R})}=\frac{C}{k}$, Estimate (26) for $\varepsilon=1$ now reads, for all $k, \mu>0$,

$$
\left\|u\left(t^{*}, \cdot\right)-M\right\|_{H^{1}(0, L)} \leq C(L+2 k) \sqrt{\frac{t^{*}}{\mu}}\|X\|_{L^{2}\left(0, t^{*} ; L^{2}(\mathbb{R})\right)} e^{\left(\frac{C}{2 k}\left\|f^{\prime \prime}\right\|_{L^{\infty}(M, N)}+\frac{\mu}{2}\right) t^{*}-\frac{1}{4 t^{*}}\left(f^{\prime}(M) t^{*}-L-2 k\right)^{2}} .
$$

Noting that $\|X\|_{L^{2}\left(0, t^{*} ; L^{2}(\mathbb{R})\right)}$ increases at most linearly in $t^{*}$ and fixing $k$ large enough and $\mu$ small enough so that $\left(\frac{C}{2 k}\left\|f^{\prime \prime}\right\|_{L^{\infty}(M, N)}+\frac{\mu}{2}\right)<\frac{f^{\prime}(M)^{2}}{4}$, we obtain the sought exponential decay as $t^{*} \rightarrow+\infty$. 


\subsection{Local exact controllability}

In this section, we perform the local controllability argument. We suppose that the initial condition $u_{0}$ is $H^{1}$-exponentially close (in terms of $\varepsilon$ ) to the constant target, say $N$, and we want to reach it exactly. This will be done both after the "shock phase" and the rarefaction phase, i.e., for an initial datum that satisfies the estimate (10) or (26). More precisely, we prove the following proposition, where we assume that $f^{\prime}(N)>0$ for simplicity (the case $f^{\prime}(N)<0$ follows the same procedure).

Proposition 2.8. Assume that $f^{\prime}(N)>0$, and that $u_{0} \in H^{1}(0, L)$ satisfies

$$
\left\|u_{0}-N\right\|_{H^{1}(0, L)} \leq e^{-\frac{K_{0}}{\varepsilon}}
$$

for some $K_{0}>0$. Then, there exist $\alpha_{1}>0$ and $\varepsilon_{0}>0$ such that for all $T \geq \alpha_{1} \frac{L}{f^{\prime}(N)}$ and $0<\varepsilon<\varepsilon_{0}$, there exist two control functions $g_{0}$ and $g_{L}$, with

$$
\left\|g_{0}\right\|_{L^{\infty}(0, T)} \leq 2|N| \quad \text { and } \quad\left\|g_{L}\right\|_{L^{\infty}(0, T)} \leq 2|N|,
$$

such that the solution $u$ of (1), (2) and (3) satisfies

$$
\left.u\right|_{t=T}=N \quad \text { in }(0, L) .
$$

The proof of Proposition 2.8 follows the steps of [15]. When doing this, we shall see that one can take $\alpha_{1}=5$ (or, as found numerically $\alpha_{1}=4.3$ ).

We first set $y(t, x)=u(t, x)-N$, so that $y$ satisfies

$$
\begin{cases}y_{t}+[f(N+y)]_{x}-\varepsilon y_{x x}=0 & \text { in }(0, T) \times(0, L), \\ \left.y\right|_{t=0}=y_{0}=u_{0}-N & \text { in }(0, L), \\ \left\|y_{0}\right\|_{H^{1}(0, L)} \leq e^{-\frac{K_{0}}{\varepsilon}} & \end{cases}
$$

Now, our objective is to find boundary controls $\left.y\right|_{x=0}=g_{0}(t)-N$ and $\left.y\right|_{x=L}=g_{L}(t)-N$ such that $\left.y\right|_{t=T}=0$ and

$$
\left\|g_{0}-N\right\|_{L^{\infty}(0, T)} \leq|N| \text { and }\left\|g_{L}-N\right\|_{L^{\infty}(0, T)} \leq|N|
$$

More precisely, we prove the existence of a controlled solution $y$, satisfying (50) and $\left.y\right|_{t=T}=0$, and then take the traces of $y$ on $(0, T) \times\{0\}$ and $(0, T) \times\{L\}$ to obtain the controls. The existence of such a controlled solution is proved by means of a fixed point argument. For this, let us first consider the following linearization of System (50), for some $z \in L^{1}\left(0, T ; W^{1, \infty}(0, L)\right) \cap L^{\infty}((0, T) \times(0, L))$ :

$$
\begin{cases}y_{t}-\varepsilon y_{x x}+[\sigma(N+z(t, x), N) y]_{x}=0 & \text { in }(0, T) \times(0, L), \\ \left.y\right|_{x=0}=\tilde{g}_{0},\left.\quad y\right|_{x=L}=\tilde{g}_{L} & \text { in }(0, T), \\ \left.y\right|_{t=0}=y_{0} & \text { in }(0, L),\end{cases}
$$

where we have denoted $\sigma(N+z, N)=\frac{f(N+z)-f(N)}{z}$, with $\sigma(N+\cdot, N) \in W_{\text {loc }}^{1, \infty}(\mathbb{R})$. Note that formally, a fixed point of a map $z \mapsto y$, where $y$ is a solution of (51) associated to some controls $\tilde{g}_{0}, \tilde{g}_{L}$, is a solution of the problem (50). It will be convenient to extend this control problem to $(a, b)$ for some $a<0$ and $b>L$, and introduce $\tilde{y}_{0}$ and $\tilde{z}$ smooth extensions of $y_{0}$ and $z$, satisfying

$$
\begin{aligned}
& \tilde{y}_{0}=y_{0} \text { on }(0, L), \quad \tilde{y}_{0}(a)=\tilde{y}_{0}(b)=0, \text { and }\left\|\tilde{y}_{0}\right\|_{H_{0}^{1}(a, b)} \leq C_{E}\left\|y_{0}\right\|_{H^{1}(0, L)}, \\
& \tilde{z}=z \text { on }(0, T) \times(0, L), \text { and }\|\tilde{z}\|_{L^{1} W^{1, \infty} \cap L^{\infty} L^{\infty}} \leq C_{E}\|z\|_{L^{1} W^{1, \infty} \cap L^{\infty} L^{\infty}} .
\end{aligned}
$$

(see for instance [12, Section 5.4]). We now consider the following extended linear system

$$
\begin{cases}\tilde{y}_{t}-\varepsilon \tilde{y}_{x x}+[\sigma(N+\tilde{z}(t, x), N) \tilde{y}]_{x}=0 & \text { in }(0, T) \times(a, b), \\ \left.\tilde{y}\right|_{x=a}=\tilde{g}(t),\left.\quad \tilde{y}\right|_{x=b}=0 & \text { in }(0, T), \\ \left.\tilde{y}\right|_{t=0}=\tilde{y}_{0} & \text { in }(a, b) .\end{cases}
$$

To prove the null-controllability of this system, we shall prove an observability estimate for its adjoint. We set

$$
\zeta(t, x)=\sigma(N+\tilde{z}(t, x), N)-f^{\prime}(N)
$$


and

$$
\lambda=b-a>L
$$

We have the following controllability lemma.

Lemma 2.9. There exist $\alpha_{1}>0$ and $\varepsilon_{0}>0$ such that for all $\zeta \in L^{1}\left(0, T ; W^{1, \infty}(0, L)\right) \cap L^{\infty}((0, T) \times$ $(0, L))$ satisfying

$$
\left\|\zeta_{x}\right\|_{L^{1} L^{\infty}}+\|\zeta\|_{L^{\infty} L^{\infty}} \leq C_{E} e^{-\frac{K_{0}}{2 \varepsilon}}
$$

(where $K_{0}$ is introduced in Proposition 2.8 and $C_{E}$ is the norm of the extension operator introduced in (52)), for all $T \geq \alpha_{1} \frac{\lambda}{f^{\prime}(N)}$, all $\varepsilon \in\left(0, \varepsilon_{0}\right)$ and $\tilde{y}_{0} \in H_{0}^{1}(a, b)$ satisfying $\left\|\tilde{y}_{0}\right\|_{H_{0}^{1}(a, b)} \leq C_{E} e^{-\frac{K_{0}}{\varepsilon}}$, there exists a control function $\tilde{g} \in L^{2}(0, T)$ with $\|\tilde{g}\|_{L^{2}(0, T)} \leq e^{-\frac{K_{0}}{\varepsilon}}$ such that the associated solution to (53) satisfies

$$
\left.\tilde{y}\right|_{t=T}=0 \quad \text { on }(a, b) .
$$

Note that the constant $\alpha_{1}$ here is the same as the one in Proposition 2.8. In the course of the proof, we shall see that one can take $\alpha_{1}$ as claimed before.

Proof. For this linear control problem (53), we use the classical approach, consisting in obtaining a suitable observability inequality for the adjoint system of (53), which reads

$$
\begin{cases}-\varphi_{t}-\varepsilon \varphi_{x x}-\left(f^{\prime}(N)+\zeta(t, x)\right) \varphi_{x}=0 & \text { in }(0, T) \times(a, b) \\ \left.\varphi\right|_{x=a}=0,\left.\quad \varphi\right|_{x=b}=0 & \text { in }(0, T) \\ \left.\varphi\right|_{t=T}=\varphi_{T} & \text { in }(a, b)\end{cases}
$$

where $\varphi_{T} \in L^{2}(a, b)$ is the final condition of this backward problem. We aim to prove the following observability inequality for the solutions of (56):

$$
\left\|\left.\varphi\right|_{t=0}\right\|_{L^{2}(a, b)} \leq K(T, \varepsilon)\left\|\left.\varphi_{x}\right|_{x=a}\right\|_{L^{2}(0, T)} .
$$

Then, classical duality arguments give the null-controllability of System (53) with a control function $\tilde{g}$ whose $L^{2}$ norm is bounded by

$$
\frac{K(T, \varepsilon)}{\varepsilon}\left\|\tilde{y}_{0}\right\|_{H_{0}^{1}(a, b)} \leq C_{E} \frac{K(T, \varepsilon)}{\varepsilon} e^{-\frac{K_{0}}{\varepsilon}} .
$$

To prove (57), we mostly follow [15] and use two of their technical estimates. More precisely, once rescaled with respect to the parameters, the dissipation estimate and the Carleman estimate read as follows $(\lambda$ is defined in (54)).

Dissipation result: for every $t \in\left(\frac{\lambda}{f^{\prime}(N)-\|\zeta\|_{L^{\infty} L^{\infty}}}, T\right)$, we have

$$
\|\varphi(0, \cdot)\|_{L^{2}(a, b)}^{2} \leq \exp \left\{\frac{\lambda^{2}\left\|\zeta_{x}\right\|_{L^{1} L^{\infty}}}{4}-\frac{\left(\left(f^{\prime}(N)-\|\zeta\|_{\left.L^{\infty} L^{\infty}\right) t}-\lambda\right)^{2}\right.}{2 \varepsilon t} e^{-4 \lambda^{2}\left\|\zeta_{x}\right\|_{L^{1} L^{\infty}}}\right\}\|\varphi(t, \cdot)\|_{L^{2}(a, b)}^{2} .
$$

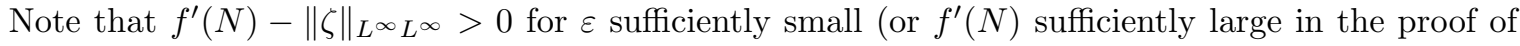
Proposition 2.11 below).

Carleman inequality: Assume that $\zeta$ satisfies (55) and $T>\frac{3}{2} \frac{\lambda}{f^{\prime}(N)-\|\zeta\|_{L^{\infty}} L^{\infty}}$. Then, we have

$$
\int_{a}^{b} \int_{2 T / 3}^{5 T / 6}|\varphi|^{2} d t d x \leq C e^{\frac{\kappa \lambda^{2}}{\varepsilon T}}\left(\int_{0}^{T}\left|\varphi_{x}(t, a)\right|^{2} d t+\int_{a}^{b}|\varphi(0, x)|^{2} d x\right) .
$$

These two estimates are proved in [15, Section 4], with $\kappa=4$ or $\kappa=2.61$ as found numerically. 
To obtain the observability inequality (57), we suppose that $T>\frac{3}{2} \frac{\lambda}{f^{\prime}(N)-\|\zeta\|_{L^{\infty} L^{\infty}}}$. As a consequence (58) holds on $\left(\frac{2 T}{3}, \frac{5 T}{6}\right) \subset\left(\frac{\lambda}{f^{\prime}(N)-\|\zeta\|_{L^{\infty} L^{\infty}}}, T\right)$. Integrating (58) on $\left(\frac{2 T}{3}, \frac{5 T}{6}\right)$, we hence obtain

$$
\begin{aligned}
\frac{T}{6}\|\varphi(0, \cdot)\|_{L^{2}(a, b)}^{2} & \leq \exp \left\{\frac{\lambda^{2}\left\|\zeta_{x}\right\|_{L^{1} L^{\infty}}}{4}\right\} \\
& \times \int_{2 T / 3}^{5 T / 6} \exp \left\{-\frac{\left(\left(f^{\prime}(N)-\|\zeta\|_{\left.\left.L^{\infty} L^{\infty}\right) t-\lambda\right)^{2}}\right.\right.}{2 \varepsilon t} e^{-4 \lambda^{2}\left\|\zeta_{x}\right\|_{L^{1} L^{\infty}}}\right\}\|\varphi(t, \cdot)\|_{L^{2}(a, b)}^{2} d t .
\end{aligned}
$$

Using the fact that

$$
t \mapsto \frac{\left(\left(f^{\prime}(N)-\|\zeta\|_{\left.L^{\infty} L^{\infty}\right) t}-\lambda\right)^{2}\right.}{2 \varepsilon t}
$$

is an increasing function as soon as $t>\frac{\lambda}{f^{\prime}(N)-\|\zeta\|_{L^{\infty} L_{L} \infty}}$, together with (59), we have

$$
\|\varphi(0, \cdot)\|_{L^{2}(a, b)}^{2} \leq C e^{-\frac{D(\varepsilon, T, \lambda, \zeta)}{\varepsilon T}}\left(\int_{0}^{T}\left|\varphi_{x}(t, a)\right|^{2} d t+\int_{a}^{b}|\varphi(0, x)|^{2} d x\right),
$$

with

$$
D(\varepsilon, T, \lambda, \zeta)=\frac{3 e^{-4 \lambda^{2}\left\|\zeta_{x}\right\|_{L^{1} L^{\infty}}}}{4}\left(\left(f^{\prime}(N)-\|\zeta\|_{L^{\infty} L^{\infty}}\right) \frac{2 T}{3}-\lambda\right)^{2}-\kappa \lambda^{2} .
$$

Now we see that for $T$ sufficiently large, i.e. $T \geq \alpha_{1} \frac{\lambda}{f^{\prime}(N)}$, we have $D(\varepsilon, T, \lambda, \zeta)>0$ and we can absorb the last term in (60) by the left hand-side, taking $\varepsilon_{0}=\varepsilon_{0}\left(\alpha_{1}\right)$ sufficiently small so that it works with $T=\alpha_{1} \frac{\lambda}{f^{\prime}(N)}$. We also notice that we can take $\alpha_{1}=5$ if $\kappa=4$ or $\alpha_{1}=4.30$ if $\kappa=2.61$. Finally, we obtain the observability inequality $(57)$ with $K(T, \varepsilon)=C e^{-\frac{D(\varepsilon, T, \lambda, \zeta)}{\varepsilon T}}$. This concludes the proof of Lemma 2.9.

Now given this result for the linearized system, we are able to implement a fixed point strategy to conclude the proof of Proposition 2.8.

Proof of Proposition 2.8. We first recall Kakutani's fixed point Theorem as presented in [23, Theorem 9.2.2].

Theorem 2.10. Let $\mathcal{Z}$ be a Banach space, $E$ a subset of $\mathcal{Z}$ and $\Lambda: E \rightarrow 2^{\mathcal{Z}}$ a multivalued mapping. Suppose that

(i) $E$ is compact convex nonempty and for every $z \in E, \Lambda(z) \subset E$.

(ii) For every $z \in E, \Lambda(z)$ is a compact convex nonempty subset of $\mathcal{Z}$.

(iii) $\Lambda$ is "upper semicontinuous", i.e. if $z_{n} \rightarrow z$ in $E$ and $y_{n} \in \Lambda\left(z_{n}\right)$ satisfies $y_{n} \rightarrow y$ in $\mathcal{Z}$, then $y \in \Lambda(z)$.

Then $\Lambda$ has a fixed point in $E$, i.e. there exists $z \in E$ such that $z \in \Lambda(z)$.

Let us now define the appropriate space $\mathcal{Z}$, subset $E$ and mapping $\Lambda$. We choose

$$
\mathcal{Z}=H^{\frac{3}{4}}\left(0, T ; L^{2}(0, L)\right) \cap L^{2}\left(0, T ; H^{1}(0, L)\right),
$$

for the Banach space and

$$
E^{\varepsilon}=\left\{z \in H^{1}\left(0, T ; L^{2}(0, L)\right) \cap L^{2}\left(0, T ; H^{2}(0, L)\right),\|z\|_{H^{1} L^{2} \cap L^{2} H^{2}} \leq e^{-\frac{K_{0}}{2 \varepsilon}}\right\} \subset \mathcal{Z} .
$$

Given a fixed $y_{0} \in H^{1}(0, L)$ such that $\left\|y_{0}\right\|_{H^{1}(0, L)} \leq e^{-\frac{K_{0}}{\varepsilon}}$, we set

$$
\Lambda(z)=\left\{y \in H^{1}\left(0, T ; L^{2}(0, L)\right) \cap L^{2}\left(0, T ; H^{2}(0, L)\right) \text { satisfying conditions (62)-(65) }\right\}
$$


with

$$
\begin{gathered}
y_{t}-\varepsilon y_{x x}=-[\sigma(N+z, N) y]_{x} \quad \text { in }(0, T) \times(0, L), \\
\left.y\right|_{t=0}=y_{0} \quad \text { in }(0, L), \\
\left.y\right|_{t=T}=0 \quad \text { in }(0, L), \\
\|y\|_{H^{1} L^{2} \cap L^{2} H^{2}} \leq e^{-\frac{K_{0}}{2 \varepsilon}},
\end{gathered}
$$

and check that Kakutani's Theorem applies with $\mathcal{Z}, E^{\varepsilon}$ and $\Lambda$ for $\varepsilon$ sufficiently small.

To prove $(i)$, note that the compact injection of $H^{1} L^{2} \cap L^{2} H^{2}$ in $\mathcal{Z}$ gives the compactness of $E^{\varepsilon}$ in $\mathcal{Z}$ and the fact that it is a ball yields its convexity. Moreover $E^{\varepsilon}$ is nonempty since it contains the null function, and $\Lambda(z) \subset E^{\varepsilon}$ if $z \in E^{\varepsilon}$, as a consequence of their definition.

To prove (ii), notice first that $\Lambda(z)$ is convex since the conditions (62)-(64) are linear and (65) is convex. To prove that it is closed (and hence compact), let us consider a sequence $\left(y_{n}\right)_{n \in \mathbb{N}} \subset \Lambda(z)$ converging to $y$ in $\mathcal{Z}$. Since $\mathcal{Z} \subset \mathcal{C}^{0}\left([0, T] ; L^{2}(0, L)\right)$, conditions (63) and (64) are still valid for the limit $y$. Since the right hand-side of $(65)$ does not depend on $n$, this estimate also holds for $y$. Moreover, $y_{n}$ converges to $y$ in $\mathcal{D}^{\prime}((0, T) \times(0, L))$ and hence, the linear equation (62) is satisfied by $y$ in $\mathcal{D}^{\prime}((0, T) \times(0, L))$. As a consequence of $(65), y \in H^{1} L^{2} \cap L^{2} H^{2}$, so that $y$ satisfies $(62)$ in $L^{2} L^{2}$, and $\Lambda(z)$ is closed.

Let us now prove that $\Lambda(z)$ is non-empty if $z \in E^{\varepsilon}$. We denote by $\tilde{z}$ and $\tilde{y}_{0}$ extensions of $z$ and $y_{0}$ on $(a, b)$ satisfying $(52)$. Denoting $\tilde{y}$ the associated solution of (53) (for any control $\tilde{g}$ ), we see that $\left.\tilde{y}\right|_{(0, L)}$ solves $(62)-(63)$. Moreover, we have $\left\|\tilde{y}_{0}\right\|_{H_{0}^{1}(a, b)} \leq C e^{-\frac{K_{0}}{\varepsilon}}$ and

$$
\|\tilde{z}\|_{L^{1} W^{1, \infty}(a, b) \cap L^{\infty} L^{\infty}(a, b)} \leq C\|\tilde{z}\|_{L^{1} W^{1, \infty}(0, L) \cap L^{\infty} L^{\infty}(0, L)} \leq C\|z\|_{H^{1} L^{2}(0, L) \cap L^{2} H^{2}(0, L)} \leq C e^{-\frac{K_{0}}{2 \varepsilon}} .
$$

Hence, denoting by $\zeta(t, x)=\sigma(N+\tilde{z}, N)-f^{\prime}(N)$, we have

$$
\|\zeta\|_{L^{\infty} L^{\infty}} \leq \frac{\left\|f^{\prime \prime}\right\|_{L^{\infty}(K)}}{2}\|\tilde{z}\|_{L^{\infty} L^{\infty}} \leq C e^{-\frac{K_{0}}{2 \varepsilon}}
$$

for some compact $K \subset \mathbb{R}$ containing $N$, and

$$
\left\|\zeta_{x}\right\|_{L^{1} L^{\infty}}=\left\|[\sigma(N+\tilde{z}, N)]_{x}\right\|_{L^{1} L^{\infty}} \leq\left\|f^{\prime \prime}\right\|_{L^{\infty}(K)}\left\|\tilde{z}_{x}\right\|_{L^{1} L^{\infty}} \leq C e^{-\frac{K_{0}}{2 \varepsilon}} .
$$

As a consequence of (66) and (67), Estimate (55) holds and Lemma 2.9 applies as soon as $T \geq \alpha_{1} \frac{\lambda}{f^{\prime}(N)}$ (that we shall suppose in the following). In particular, for $\varepsilon<\varepsilon_{0}$, there exists a control function $\tilde{g}$ such that $\|\tilde{g}\|_{L^{2}(0, T)} \leq C e^{-\frac{K_{0}}{\varepsilon}}$ and the associated solution of (53) satisfies $\left.\tilde{y}\right|_{t=T}=0$, and thus $\left.\tilde{y}\right|_{(0, L)}$ fulfills (64). Moreover, $\tilde{y}$ is defined as a transposition solution of (53) so that the regularity estimate (84) of Lemma 4.2 gives, for some $C>0$ independent from $\varepsilon$,

$$
\|\tilde{y}\|_{L^{2} L^{2}(a, b)} \leq \frac{C}{\sqrt{\varepsilon}}\left(\|\tilde{g}\|_{L^{2}(0, T)}+\left\|\tilde{y}_{0}\right\|_{L^{2}(a, b)}\right) .
$$

We now take open intervals $\Omega_{1}$ and $\Omega_{2}$ such that $[0, L] \subset \Omega_{2} \subset \bar{\Omega}_{2} \subset \Omega_{1} \subset \bar{\Omega}_{1} \subset(a, b)$ and a cut-off function $\chi_{1} \in \mathcal{C}_{c}^{\infty}\left(\Omega_{1}\right)$ defined as before. The function $w_{1}=\chi_{1} \tilde{y}$ satisfies

$$
\begin{cases}w_{1, t}-\varepsilon w_{1, x x}=-\chi_{1}[\sigma(N+\tilde{z}, N) \tilde{y}]_{x}-2 \varepsilon \chi_{1}^{\prime} \tilde{y}_{x}-\varepsilon \chi_{1}^{\prime \prime} \tilde{y} & \text { in }(0, T) \times \Omega_{1} \\ w_{1}=0, & \text { in }(0, T) \times \partial \Omega_{1}, \\ \left.w_{1}\right|_{t=0}=\chi_{1} \tilde{y}_{0} & \in H_{0}^{1}\left(\Omega_{1}\right)\end{cases}
$$

so that the parabolic regularity result of Lemma 4.1, taken for $m=0$, gives

$$
\left\|w_{1}\right\|_{L^{2} H_{0}^{1}\left(\Omega_{1}\right)} \leq \frac{C}{\varepsilon}\left(\left\|\chi_{1} \tilde{y}_{0}\right\|_{L^{2}\left(\Omega_{1}\right)}+\left\|\chi_{1}[\sigma(N+\tilde{z}, N) \tilde{y}]_{x}+2 \varepsilon \chi_{1}^{\prime} \tilde{y}_{x}+\varepsilon \chi_{1}^{\prime \prime} \tilde{y}\right\|_{L^{2} H^{-1}\left(\Omega_{1}\right)}\right) .
$$


The last two terms in the right hand-side can be estimated by $C\|\tilde{y}\|_{L^{2} L^{2}\left(\Omega_{1}\right)}$, and we have

$$
\left\|\chi_{1}[\sigma(N+\tilde{z}, N) \tilde{y}]_{x}\right\|_{L^{2} H^{-1}\left(\Omega_{1}\right)} \leq C\|\sigma(N+\tilde{z}, N) \tilde{y}\|_{L^{2} L^{2}\left(\Omega_{1}\right)} \leq C\|\tilde{y}\|_{L^{2} L^{2}(a, b)}
$$

since $\sigma(N+\cdot, N) \in L^{\infty}$ and $\tilde{z} \in L^{\infty} L^{\infty}$. As a consequence of (68), we now obtain

$$
\left\|w_{1}\right\|_{H^{1} H^{-1}\left(\Omega_{1}\right) \cap L^{2} H_{0}^{1}\left(\Omega_{1}\right) \cap L^{\infty} L^{2}\left(\Omega_{1}\right)} \leq \frac{C}{\varepsilon^{\frac{3}{2}}}\left(\|\tilde{g}\|_{L^{2}(0, T)}+\left\|\tilde{y}_{0}\right\|_{L^{2}(a, b)}\right) .
$$

Taking another cut-off function $\chi_{2} \in \mathcal{C}_{c}^{\infty}\left(\Omega_{2}\right)$ as before, the function $w_{2}=\chi_{2} w_{1}=\chi_{2} \tilde{y}$ satisfies

$$
\begin{cases}w_{2, t}-\varepsilon w_{2, x x}=-\chi_{2}\left[\sigma(N+\tilde{z}, N) w_{1}\right]_{x}-2 \varepsilon \chi_{2}^{\prime} w_{1, x}-\varepsilon \chi_{2}^{\prime \prime} w_{1} & \text { in }(0, T) \times \Omega_{2}, \\ w_{2}=0, & \text { in }(0, T) \times \partial \Omega_{2}, \\ \left.w_{2}\right|_{t=0}=\chi_{2} w_{1} & \in H_{0}^{1}\left(\Omega_{2}\right),\end{cases}
$$

so that the parabolic regularity result of Lemma 4.1, taken for $m=1$, gives

$$
\begin{aligned}
\left\|w_{2}\right\|_{L^{2}\left(H^{2} \cap H_{0}^{1}\left(\Omega_{2}\right)\right) \cap H^{1} L^{2}\left(\Omega_{2}\right)} \leq \frac{C}{\varepsilon}( & \left\|\chi_{2} w_{1}\right\|_{H_{0}^{1}\left(\Omega_{2}\right)} \\
& \left.+\left\|\chi_{2}\left[\sigma(N+\tilde{z}, N) w_{1}\right]_{x}+2 \varepsilon \chi_{2}^{\prime} w_{0, x}+\varepsilon \chi_{2}^{\prime \prime} w_{1}\right\|_{L^{2} L^{2}\left(\Omega_{2}\right)}\right) .
\end{aligned}
$$

In this expression, we can estimate

$$
\begin{aligned}
\left\|\chi_{2}\left[\sigma(N+\tilde{z}, N) w_{1}\right]_{x}\right\|_{L^{2} L^{2}\left(\Omega_{2}\right)} & \leq\left\|\sigma(N+\tilde{z}, N) w_{0, x}\right\|_{L^{2} L^{2}\left(\Omega_{1}\right)}+\left\|[\sigma(N+\tilde{z}, N)]_{x} w_{1}\right\|_{L^{2} L^{2}\left(\Omega_{1}\right)} \\
& \leq\|\sigma(N+\tilde{z}, N)\|_{L^{\infty} L^{\infty}}\left\|w_{1}\right\|_{L^{2} H_{0}^{1}\left(\Omega_{1}\right)}+\|\sigma(N+\tilde{z}, N)\|_{L^{2} W^{1, \infty}}\left\|w_{1}\right\|_{L^{\infty} L^{2}\left(\Omega_{1}\right)}
\end{aligned}
$$

where all the terms in the right hand-side are finite, since $\sigma(N+\cdot, N) \in L^{\infty}, \tilde{z} \in L^{\infty} L^{\infty} \cap L^{2} W^{1, \infty}$, and $w_{1}$ satisfies (69). Replacing this in (70) and using (69) yields

$$
\left\|w_{2}\right\|_{L^{2}\left(H^{2} \cap H_{0}^{1}\left(\Omega_{2}\right)\right) \cap H^{1} L^{2}\left(\Omega_{2}\right)} \leq \frac{C}{\varepsilon^{\frac{5}{2}}}\left(\|\tilde{g}\|_{L^{2}(0, T)}+\left\|\tilde{y}_{0}\right\|_{L^{2}(a, b)}\right),
$$

and thus

$$
\left\|\left.\tilde{y}\right|_{(0, L)}\right\|_{L^{2} H^{2}(0, L) \cap H^{1} L^{2}(0, L)} \leq\left\|w_{2}\right\|_{L^{2}\left(H^{2} \cap H_{0}^{1}\left(\Omega_{2}\right)\right) \cap H^{1} L^{2}\left(\Omega_{2}\right)} \leq \frac{C}{\varepsilon^{\frac{5}{2}}}\left(2 e^{-\frac{K_{0}}{\varepsilon}}+e^{-\frac{K_{0}}{\varepsilon}}\right) \leq e^{-\frac{K_{0}}{2 \varepsilon}},
$$

for $0<\varepsilon<\varepsilon_{0}$. This implies that $\left.\tilde{y}\right|_{(0, L)}$ satisfies (65) for $0<\varepsilon<\varepsilon_{0}$ and hence all conditions (62)-(65), and $\Lambda(z)$ is nonempty.

To prove (iii), let us consider two sequences, $z_{n} \rightarrow z$ in $E^{\varepsilon}$ for the topology of $H^{1} L^{2} \cap L^{2} H^{2}$, and $y_{n} \in \Lambda\left(z_{n}\right)$ satisfying $y_{n} \rightarrow y$ in $\mathcal{Z}$, and check that $y \in \Lambda(z)$. The three assertions (63)-(65) still hold for $y$ since (63)-(65) do not depend on $z_{n}$, and in particular $y \in H^{1} L^{2} \cap L^{2} H^{2}$. Let us denote $R_{n}$ the right hand-side of (62) for $z_{n}$ and $y_{n}$, and $R$ its counterpart for $z$ and $y$. We have

$$
R_{n}-R=[\sigma(N+z, N)]_{x} y+\sigma(N+z, N) y_{x}-\left[\sigma\left(N+z_{n}, N\right)\right]_{x} y_{n}-\sigma\left(N+z_{n}, N\right) y_{n, x}
$$

and hence $\left\|R_{n}-R\right\|_{L^{2} L^{2}} \leq A_{n}+B_{n}$, with

$$
\begin{aligned}
& A_{n}=\left\|[\sigma(N+z, N)]_{x} y-[\sigma(N+z, N)]_{x} y_{n}\right\|_{L^{2} L^{2}}+\left\|[\sigma(N+z, N)]_{x} y_{n}-\left[\sigma\left(N+z_{n}, N\right)\right]_{x} y_{n}\right\|_{L^{2} L^{2}} \\
& \quad \leq\left\|[\sigma(N+z, N)]_{x}\right\|_{L^{2} L^{\infty}}\left\|y-y_{n}\right\|_{L^{\infty} L^{2}}+\left\|[\sigma(N+z, N)]_{x}-\left[\sigma\left(N+z_{n}, N\right)\right]_{x}\right\|_{L^{2} L^{\infty}}\left\|y_{n}\right\|_{L^{\infty} L^{2}},
\end{aligned}
$$

and

$$
\begin{aligned}
B_{n}= & \left\|\sigma(N+z, N) y_{x}-\sigma(N+z, N) y_{n, x}\right\|_{L^{2} L^{2}}+\left\|\sigma(N+z, N) y_{n, x}-\sigma\left(N+z_{n}, N\right) y_{n, x}\right\|_{L^{2} L^{2}} \\
& \leq\|\sigma(N+z, N)\|_{L^{\infty} L^{\infty}}\left\|y_{x}-y_{n, x}\right\|_{L^{2} L^{2}}+\left\|\sigma(N+z, N)-\sigma\left(N+z_{n}, N\right)\right\|_{L^{\infty} L^{\infty}}\left\|y_{n, x}\right\|_{L^{2} L^{2}}
\end{aligned}
$$


Recalling that $z_{n} \rightarrow z$ in $E^{\varepsilon}$ and $y_{n} \rightarrow y$ in $\mathcal{Z}$, we deduce $\lim _{n \rightarrow+\infty} A_{n}=0$ since $y_{n} \rightarrow y$ in $H^{\frac{3}{4}} L^{2} \subset L^{\infty} L^{2}$ and $\sigma\left(N+z_{n}, N\right) \rightarrow \sigma(N+z, N)$ in $L^{2} W^{1, \infty}$. Moreover, $\lim _{n \rightarrow+\infty} B_{n}=0$ since $y_{n} \rightarrow y$ in $L^{2} H^{1}$ and $\sigma\left(N+z_{n}, N\right) \rightarrow \sigma(N+z, N)$ in $L^{\infty} L^{\infty}$. We can now write (62) for $z_{n}$ and $y_{n}$ as

$$
y_{n, t}-\varepsilon y_{n, x x}=R_{n}
$$

where the right hand-side $R_{n}$ satisfies $R_{n} \rightarrow R$ in $L^{2} L^{2}$. This, together with (65) implies that when $n \rightarrow+\infty$ we have,

$$
y_{t}-\varepsilon y_{x x}=R \quad \text { in } L^{2} L^{2},
$$

so that (62) holds for the limit $y$. Finally, $y \in \Lambda(z)$, and condition (iii) is fulfilled.

Consequently, Kakutani's Theorem applies and there exists $y \in \Lambda(y)$, that is to say, for $y_{0}$ satisfying $\left\|y_{0}\right\|_{H^{1}(0, L)} \leq e^{-\frac{K_{0}}{\varepsilon}}$, we have found a function $y$ satisfying

$$
\begin{cases}y_{t}-\varepsilon y_{x x}+[\sigma(N+y, N) y]_{x}=0 & \text { in }(0, T) \times(0, L), \\ \left.y\right|_{t=0}=y_{0} & \text { in }(0, L), \\ \left.y\right|_{t=T}=0 & \text { in }(0, L), \\ \|y\|_{H^{1} L^{2} \cap L^{2} H^{2}} \leq e^{-\frac{K_{0}}{2 \varepsilon}} & \end{cases}
$$

It suffices now to take the control functions

$$
g_{0}(t):=\left.y\right|_{x=0}(t)+N \quad \text { and } \quad g_{L}(t):=\left.y\right|_{x=L}(t)+N .
$$

With this choice, we have $g_{0}, g_{L} \in L^{\infty}(0, T)$ since $H^{1} L^{2} \cap L^{2} H^{2} \subset H^{\frac{3}{5}} H^{\frac{4}{5}}$ and

$$
\max \left(\left\|g_{0}\right\|_{L^{\infty}(0, T)},\left\|g_{L}\right\|_{L^{\infty}(0, T)}\right) \leq|N|+C e^{-\frac{K_{0}}{2 \varepsilon}} \leq 2|N|
$$

for $0<\varepsilon<\varepsilon_{0}$. In addition, this proof works for any time of control $T \geq \alpha_{1} \frac{\lambda}{f^{\prime}(N)}$, for any $\lambda>L$ (but all the constants depend on $\lambda$ ). This yields the result for any $T \geq \alpha_{1} \frac{L}{f^{\prime}(N)}$, and concludes the proof of Proposition 2.8.

To conclude this section, we give a slight modification of Proposition 2.8, adapted to the first phase of the proof of Theorem 1.1, which consists in reaching exactly a large state $N$, without assuming $\varepsilon$ small. To this aim, the strategy adopted here is the same as the one of Proposition 2.8 except that we take " $N$ large" instead of " $\varepsilon$ small" in the fixed point argument. To do so, we carefully keep track of the dependence with respect to the parameter $N$, as $N \rightarrow+\infty$.

Proposition 2.11. Suppose that Assumption $(\mathrm{A}+)$ holds. Then, there exist $\alpha_{1}>0$ such that for all $\varepsilon_{0}>0$, there exist $N_{0}>0$ such that for all $\varepsilon \in\left(0, \varepsilon_{0}\right), N>N_{0}, T \geq \alpha_{1} \frac{L}{f^{\prime}(N)}$, and $u_{0} \in H^{1}(0, L)$ satisfying for some $P<N_{0}$

$$
\left\|u_{0}-N\right\|_{H^{1}(0, L)} \leq C(N-P)\left\|f^{\prime}\right\|_{L^{\infty}(P, N)}^{\frac{5}{2}}\left(\left\|f^{\prime}\right\|_{L^{\infty}(P, N)}+\left\|f^{\prime \prime}\right\|_{L^{\infty}(P, N)}\|f\|_{L^{\infty}(P, N)}\right) e^{-K_{0} s\left(\sigma_{m}-s\right)},
$$

with $s=\sigma(P, N), \sigma_{m}=\sigma(E, N)$ and $P<E$, there exist two control functions $g_{0}$ and $g_{L}$ such that the result of Proposition 2.8 holds.

To use this after Proposition 2.1, we take $E=\operatorname{ess} \inf u_{0}>P$. We now sketch the proof of this proposition.

Proof. Here, we only replace the argument " $\varepsilon$ small" in the previous proof by " $N$ large". This is possible since Assumption (A+) implies the existence of $Q \in \mathbb{R}, l_{0}, C>0, \gamma>-\frac{1}{2}$ and $\delta>0$ such that

$$
f^{\prime \prime}(A) \geq l_{0} A^{\gamma} \quad \text { and } \quad f^{\prime \prime}(A) \leq C e^{A^{2 \gamma+1-\delta}}
$$

for all $A>Q$. Since we have

$$
f(N)=f(Q)+(N-Q) f^{\prime}(Q)+\int_{Q}^{N} f^{\prime \prime}(\tau)(N-\tau) d \tau \geq \frac{l_{0}}{(\gamma+1)(\gamma+2)} N^{\gamma+2}+O(N),
$$


we can estimate

$$
\sigma_{m}-s \geq(E-P) \frac{f(N)}{N^{2}}+O\left(\frac{1}{N}\right) \geq(E-P) \frac{l_{0}}{(\gamma+1)(\gamma+2)} N^{\gamma}+O\left(\frac{1}{N}\right)
$$

and

$$
s \geq(E-P) \frac{f(N)}{2 N}+O\left(\frac{1}{N}\right) \geq \frac{l_{0}}{2(\gamma+1)(\gamma+2)} N^{\gamma+1}+O(1),
$$

so that

$$
e^{-K_{0} s\left(\sigma_{m}-s\right)} \leq C e^{-\frac{K_{0} l_{0}^{2}(E-P)}{2(\gamma+1)^{2}(\gamma+2)^{2}} N^{2 \gamma+1}} .
$$

Since we supposed $\gamma>-\frac{1}{2}$, this expression is exponentially decaying as $N \rightarrow+\infty$. It sufficies now to follow the proof of Proposition 2.8, replacing the exponential decay $\left\|u_{0}-N\right\|_{H^{1}(0, L)} \leq e^{-\frac{K_{0}}{\varepsilon}}$ as $\varepsilon \rightarrow 0^{+}$, by the exponential decay

$$
\begin{aligned}
\left\|u_{0}-N\right\|_{H^{1}(0, L)} & \leq C(N-P)\left\|f^{\prime}\right\|_{L^{\infty}(P, N)}^{\frac{5}{2}}\left(\left\|f^{\prime}\right\|_{L^{\infty}(P, N)}+\left\|f^{\prime \prime}\right\|_{L^{\infty}(P, N)}\|f\|_{L^{\infty}(P, N)}\right) e^{-K_{0} s\left(\sigma_{m}-s\right)} \\
& \leq C e^{N^{2 \gamma+1-\delta}} e^{-\frac{K_{0} l_{0}^{2}(E-P)}{2(\gamma+1)^{2}(\gamma+2)^{2}} N^{2 \gamma+1}} \leq e^{-K_{1} N^{2 \gamma+1}}
\end{aligned}
$$

as $N \rightarrow+\infty$, for some $K_{1}>0$, as a consequence of Assumption $(\mathrm{A}+)$ and (74). We also have to use systematically $e^{-\frac{K_{1}}{2} N^{2 \gamma+1}}$ in place of $e^{-\frac{K_{0}}{2 \varepsilon}}$ and $N>N_{0}$ in place of $\varepsilon<\varepsilon_{0}$ in the proof Proposition 2.8. The analogous of Estimates (66) and (67) are performed using the fact that in these expressions, $K$ is the ball centered on $N$ of radius smaller than $e^{-K_{1} N^{2 \gamma+1}}$.

Remark 2.12. This is the only place where we use the unnatural assumption $(A+)$. Any other condition on $f$ allowing to perform the fixed point argument is sufficient for proving Theorem 1.1. Note that if we had proved a decay rate of the form $\theta s\left(f^{\prime}(N)-s\right)$, Estimate (74) would be replaced by $e^{-K_{0} s\left(f^{\prime}(N)-s\right)} \leq C e^{-C N^{2 \gamma+2}}$, which explains the more general condition written in Remark 2.3 .

\section{Proofs of the three theorems}

With the use of Propositions 2.1, 2.4 and 2.8 (resp. 2.11), we are now able to prove Theorems $1.2,1.3$ (resp. 1.1). Before that, we combine the global approximate controllability results and the local one to provide two different global exact controllability results using a traveling wave (see Proposition 3.1 below) or a rarefaction wave (see Proposition 3.2 below). After that, we can conclude the proofs of the theorems.

Proposition 3.1. Suppose that there exist constant states $P$ and $N$, such that one of these conditions is satisfied:

(a) $P<\operatorname{ess} \inf u_{0} \leq \operatorname{ess} \sup u_{0}<N, f(N)>f(P)$, and (SOC+) holds on $(P, N)$;

(b) $N<$ ess inf $u_{0} \leq$ ess $\sup u_{0}<P, f(N)>f(P)$, and (SOC+) holds on $(N, P)$;

(c) $P<\operatorname{ess} \inf u_{0} \leq \operatorname{ess} \sup u_{0}<N, f(N)<f(P)$, and (SOC-) holds on $(P, N)$;

(d) $N<$ ess inf $u_{0} \leq$ ess sup $u_{0}<P, f(N)<f(P)$, and (SOC-) holds on $(N, P)$.

Then, setting $s=\sigma(P, N)$, there exist $C>0$ only depending on $f$ and $\varepsilon_{0}>0$ such that for all $T>\alpha_{1} \frac{L}{\left|f^{\prime}(N)\right|}+\frac{L}{|s|}$ and $0<\varepsilon<\varepsilon_{0}$, there exist two control functions $g_{0}$ and $g_{L}$, with

$$
\left\|g_{0}\right\|_{L^{\infty}(0, T)} \leq C(|N|+|P|) \quad \text { and } \quad\left\|g_{L}\right\|_{L^{\infty}(0, T)} \leq C(|N|+|P|),
$$

such that the solution $u$ of (1), (2) and (3) satisfies

$$
\left.u\right|_{t=T}=N \quad \text { in }(0, L) \text {. }
$$


Proof. Here, we only prove the proposition in the case $(a)$. The proof of cases $(b)-(d)$ follows the one of $(a)$ for a modified Proposition 2.1, since the conditions that we write are the conditions of existence of a traveling wave from $P$ to $N$ or from $N$ to $P$.

Since $P$ and $N$ satisfy the strict condition (SOC+) and $f(N)>f(P)$, one can find $P^{\prime}$ close to $P$, and $N^{\prime}$ close to $N$, such that the stronger condition (POC) is satisfied on $\left(P^{\prime}, N^{\prime}\right)$, together with $P^{\prime}<\operatorname{ess} \inf u_{0} \leq \operatorname{ess} \sup u_{0}<N^{\prime}$ and $f\left(N^{\prime}\right)>f\left(P^{\prime}\right)$. As a consequence, Proposition 2.1 applies and hence one can find control functions that drive $u_{0}$ to a state $u_{1}$ which is $H^{1}$-exponentially close to $N$ in any time $T_{1}>\frac{L}{|s|}$. After that, the assumptions of Proposition 2.8 are satisfied, and thus $u_{1}$ can be steered exacly to $N$ in any time $T_{2} \geq \alpha_{1} \frac{L}{\left|f^{\prime}(N)\right|}$. Finally the result of Proposition 3.1 holds for any time $T \geq T_{1}+T_{2}$.

Proposition 3.2. Suppose that one of these conditions is satisfied:

(a) $M<N, f^{\prime \prime} \geq 0$ on the interval $(M, N)$ and $f^{\prime}(M)>0$;

(b) $N<M, f^{\prime \prime} \geq 0$ on the interval $(N, M)$ and $f^{\prime}(M)<0$;

(c) $M<N, f^{\prime \prime} \leq 0$ on the interval $(M, N)$ and $f^{\prime}(M)<0$;

(d) $N<M, f^{\prime \prime} \leq 0$ on the interval $(N, M)$ and $f^{\prime}(M)>0$.

Then, there exist $C>0$ only depending on $f$ and $\varepsilon_{0}>0$ such that for all $T>\left(\alpha_{1}+1\right) \frac{L}{\left|f^{\prime}(M)\right|}$ and $0<\varepsilon<\varepsilon_{0}$, there exist two control functions $g_{0}$ and $g_{L}$, with

$$
\left\|g_{0}\right\|_{L^{\infty}(0, T)} \leq C(|M|+|N|) \quad \text { and } \quad\left\|g_{L}\right\|_{L^{\infty}(0, T)} \leq C(|M|+|N|),
$$

such that the solution $u$ of (1), (2) with initial condition $\left.u\right|_{t=0}=N$ satisfies,

$$
\left.u\right|_{t=T}=M \quad \text { in }(0, L) .
$$

Proof. Here, we only prove the proposition in the case $(a)$. The proof of cases $(b)-(d)$ follows the one of $(a)$ for a modified Proposition 2.4, since the conditions we write are the conditions of existence of a rarefaction wave steering $N$ to $M$, having a speed of non-vanishing fixed sign.

In the case $(a)$, the assumptions of Proposition 2.4 are satisfied and hence one can find control functions that drive $u_{0}$ to a state $u_{1}$ which is $H^{1}$-exponentially close to $M$ in any time $T_{1}>\frac{L}{\left|f^{\prime}(M)\right|}$. After that, the assumptions of Proposition 2.8 are satisfied, and thus $u_{1}$ can be steered exacly to $N$ in a time $T_{2} \geq \alpha_{1} \frac{L}{\left|f^{\prime}(M)\right|}$. Finally, the result of Proposition 3.1 holds for any time $T \geq T_{1}+T_{2}$.

\subsection{Proof of Theorems 1.1 and 1.2, the convex case}

In this section, we suppose $f^{\prime \prime} \geq 0$. Let us first prove Theorem 1.2, using Propositions 3.1 and 3.2.

Proof of Theorem 1.2. We first suppose that $f^{\prime}(M)>0$ and choose some $P<\operatorname{ess} \inf u_{0}$. Since $\lim _{u \rightarrow+\infty} f^{\prime}(u)=+\infty$, and setting $s=\sigma(N, P)$, we have $\lim _{N \rightarrow+\infty} s=+\infty$. As a consequence, for any time $T_{1}>0$ there exists $N>\operatorname{ess} \sup u_{0}$ sufficiently large so that $f(N)>f(P)$ and $0<$ $\alpha_{1} \frac{L}{f^{\prime}(N)}+\frac{L}{s}<T_{1}$. As a consequence of Proposition 3.1, one can reach $N$ exactly, uniformly with $\varepsilon$ in time $T_{1}$. Then Proposition 3.2 holds between $N$ and $M$, and for any $T_{2}>\alpha_{0} \frac{L}{f^{\prime}(M)}$ (with $\left.\alpha_{0}=\alpha_{1}+1\right)$, there exist two control functions bounded uniformly with respect to $\varepsilon$ such that $\left.u\right|_{t=T_{1}+T_{2}}=M$. Finally, the result of Theorem 1.1 holds for any time $T \geq T_{1}+T_{2}$.

In the case $f^{\prime}(M)<0$, exactly the same proof still holds using $\lim _{u \rightarrow-\infty} f^{\prime}(u)=-\infty$, together with Proposition $3.1(b)$ and Proposition $3.2(b)$.

In Theorem 1.1, however, we do not want $\varepsilon_{0}$ to depend on the initial datum $u_{0}$. For this, we use the following proposition instead of Proposition 3.1. 
Proposition 3.3. Suppose that condition $(\mathrm{A}+)$ holds. Then, for all $\varepsilon_{0}>0$ and $T>0$, there exists a state $N_{0}>0$, such that for all $\varepsilon \in\left(0, \varepsilon_{0}\right)$ and $N>N_{0}$, there exist control functions $g_{0}$ and $g_{L}$ with

$$
\left\|g_{0}\right\|_{L^{\infty}(0, T)} \leq\left\|u_{0}\right\|_{L^{\infty}(0, L)}+2|N| \quad \text { and } \quad\left\|g_{L}\right\|_{L^{\infty}(0, T)} \leq\left\|u_{0}\right\|_{L^{\infty}(0, L)}+2|N|,
$$

such that the solution $u$ of (1), (2) and (3) satisfies

$$
\left.u\right|_{t=T}=N \quad \text { in }(0, L) .
$$

The proof of this proposition combines the approximate controllability result to the large state $N$ of Proposition 2.1 and the local exact controllability result of Proposition 2.11. Both results hold for any $\varepsilon>0$, for $N$ sufficiently large, for control times $T>\frac{L}{s}$ and $T \geq \alpha_{1} \frac{L}{f^{\prime}(N)}$, both vanishing as $N \rightarrow+\infty$.

The proof of Theorem 1.1 then exactly follows that of Theorem 1.2, using both Proposition 3.3 to reach the large constant $N$ and Proposition 3.2 to come back to the state $M$ with a rarefaction wave.

\subsection{Proof of Theorem 1.3, the non-convex case}

For the sake of brevity in the following proof, we do not mention that every control step is done uniformly with respect to $\varepsilon$, as a consequence of Propositions 3.1 and 3.2.

Proof of Theorem 1.3. The first step is to "get rid of" the initial condition $u_{0}$, and that is the only role of Assumption (ii). This assumption, together with Proposition 3.1 gives the controllability from $u_{0}$ to some constant state $M_{0}$ with $f^{\prime}\left(M_{0}\right) \neq 0$. All the proof is reduced now to a controllability problem between two constant states $\left(M_{0}\right.$ and $\left.M\right)$ and is illustrated in Figure 3.

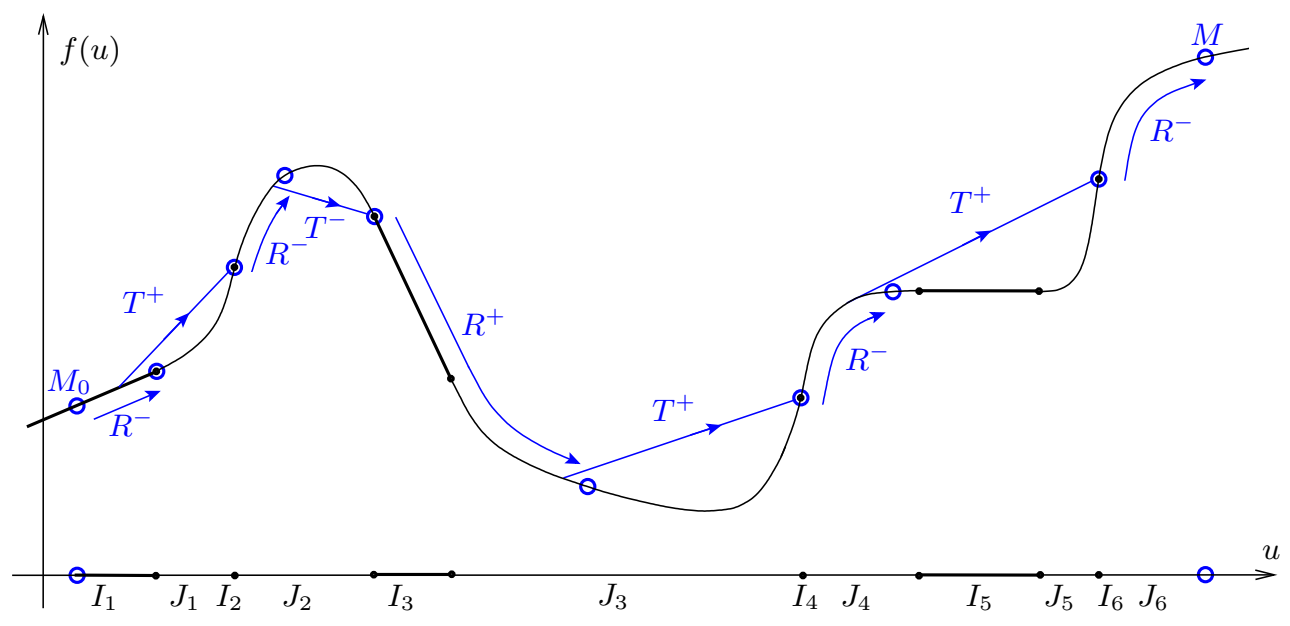

Figure 3: Global control strategy for non-convex $f$ $R^{+}$: "convex rarefaction wave"; $R^{-}$: "concave rarefaction wave"; $T^{+}$: "convex traveling wave"; $T^{-}$: "concave traveling wave".

Let us suppose that $M_{0}<M$ (the case $M_{0}>M$ follows from the same arguments). From Assumption $(i),\left(f^{\prime \prime}\right)^{-1}(\{0\}) \cap\left[M_{0}, M\right]$ can be written as $\bigcup_{j=1}^{m} I_{j}$ where the union is disjoint and ordered. Note that each $I_{j}$ is a segment (possibly reduced to a point) since $f^{\prime \prime}$ is supposed to be continuous here. Let us denote $\left(J_{k}\right)_{1 \leq k \leq p}$ the connected components of $\left(M_{0}, M\right) \backslash \bigcup_{j=1}^{m} I_{j}$, so that $\left(M_{0}, M\right) \backslash \bigcup_{j=1}^{m} I_{j}=\bigcup_{k=1}^{p} J_{k}$, where this last union is disjoint and ordered. As a consequence, $J_{k}$ is an open interval on which either $f^{\prime \prime}>0$ or $f^{\prime \prime}<0$. 
1. Driving $A$ to $B$ with $A, B \in \bar{J}_{k}$.

We first prove that, for any $A \in \bar{J}_{k}$ and $B \in \bar{J}_{k}$ satisfying $f^{\prime}(A) \neq 0$ and $f^{\prime}(B) \neq 0$, one can steer $A$ to $B$ in a finite time. We suppose that $f^{\prime \prime}>0$ on $J_{k}$ (the case $f^{\prime \prime}<0$ follows from the same arguments) and denote by $L_{k}$ (resp. $R_{k}$ ) the left (resp. right) endpoint of $\bar{J}_{k}$.

- If $f^{\prime}\left(L_{k}\right)>0$, then, as a consequence of Lemma 3.4 below, and using the fact that $f$ is strictly convex on $\left(L_{k}, R_{k}\right)$, there exists $L_{k}^{\prime}<L_{k}$ such that $f$ satisfies (SOC+) on $\left(L_{k}^{\prime}, R_{k}\right)$. Thus, there exists a traveling wave from $L_{k}^{\prime}$ to $R_{k}$ and, according to Proposition $3.1(a), A$ can be driven to $R_{k}$ in finite time (see the situation in $J_{1}$ in Figure 3 ). Then, we are in the situation of Proposition 3.2 (a) and a rarefaction wave drives $R_{k}$ to $B$ in finite time. Note that if $f^{\prime}\left(L_{k}\right)=0$, we have $L_{k}<A$ and the same strategy holds.

- If $f^{\prime}\left(R_{k}\right) \leq 0, f$ has no local minimum on $J_{k}$ and this case follows the same.

- If $f^{\prime}\left(L_{k}\right)<0$ and $f^{\prime}\left(R_{k}\right)>0$, there exists $C_{k} \in J_{k}$ satisfying $f^{\prime}\left(C_{k}\right)=0$. The situation $f^{\prime}(A) f^{\prime}(B)>0$ has already been treated, so we only have to consider the case $f^{\prime}(A)<0$ and $f^{\prime}(B)>0$ (the case $f^{\prime}(A)>0$ and $f^{\prime}(B)<0$ follows exactly the same arguments). According to Proposition $3.2(b), A$ can be brought to $A^{\prime}$ as close to $C_{k}$ as needed, by means of a rarefaction wave (see the situation in $J_{3}$ in Figure 3). It suffices to take $A^{\prime}$ so that $f\left(A^{\prime}\right)<f(B)$, and as a consequence of Proposition $3.1(a)$, we can steer $A^{\prime}$ to $B$ thanks to a traveling wave ( $f$ is convex on $J_{k}$ and hence satisfies $(\mathrm{SOC}+)$ on this interval).

2. Driving $A$ to $B$ with $A, B \in I_{j}$.

Next, we prove that, if $f^{\prime} \neq 0$ on $I_{j}$ for some $j$, then for any $A, B \in I_{j}$, one can steer $A$ to $B$ in finite time. Recall that $f^{\prime \prime}=0$ on $I_{j}$. If $f^{\prime}>0$ on $I_{j}$ and $B<A$, we are in the context of Proposition 3.2 $(a)$ and a "convex rarefaction wave" drives $A$ to $B$ (see the situation in $I_{3}$ in Figure 3 ). If $f^{\prime}>0$ on $I_{j}$ and $A<B$, Proposition $3.2(d)$ applies and a "concave rarefaction wave" drives $A$ to $B$ (see the situation in $I_{1}$ in Figure 3). We can do the same if $f^{\prime}<0$ on $I_{j}$ using Proposition $3.2(b)$ and $(c)$. Note that in this case, Equation (1) is only a linear transport equation with constant speed, which we control on both endpoints of the interval, and the result is also a consequence of [9].

3. Driving $A$ to $B$ with $A \in J_{k}$ and $B \in J_{k+1}$, when $f^{\prime}$ vanishes between $J_{k}$ and $J_{k+1}$ The last case to consider is the case where $J_{k}=\left(L_{k}, L_{j}\right), I_{j}=\left[L_{j}, R_{j}\right], J_{k+1}=\left(R_{j}, R_{k+1}\right), f^{\prime}=0$ on $I_{j}, A \in J_{k}, B \in J_{k+1}$, and we want to steer $A$ to $B$. Suppose that $f^{\prime}(A)>0$ (the case $f^{\prime}(A)<0$ follows the same). Then, according to Proposition $3.2(d)$, a "concave rarefaction wave" can bring $A$ to some $A^{\prime}$ as close as we want from $L_{j}$ (see the situation in $J_{4}$ in Figure 3 ).

- If $f^{\prime}(B)<0$, we can choose $A^{\prime}<L_{j}$ such that $f\left(A^{\prime}\right)>f(B)$, so that Proposition $3.1(c)$ holds and we can steer $A^{\prime}$ to $B$ by means of a concave traveling wave.

- If $f^{\prime}(B)>0$, the continuity of $f^{\prime}$ yields that we can choose $A^{\prime}<L_{j}$ such that $0<f^{\prime}\left(A^{\prime}\right)<$ $\sigma\left(A^{\prime}, B\right)$ so that $(\mathrm{SOC}+)$ holds on $\left(A^{\prime}, B\right)$ and, according to Proposition $3.1(a)$, we can steer $A^{\prime}$ to $B$ by means of a "convex traveling wave" (see the situation in $I_{5}$ in Figure 3 ).

Therefore, using iteratively the three arguments above, one can pass from each zone $I_{j}$ to the neighboring zone $J_{k}$ (case 2.) and to each $J_{k}$ to the neighboring $I_{j}$ (case 1.) since $I_{j} \cap \bar{J}_{k} \neq \emptyset$, provided that $f^{\prime} \neq 0$ on $I_{j}$. If $f^{\prime}=0$ on $I_{j}$, one can jump from $J_{k}$ before $I_{j}$ to $J_{k+1}$ after $I_{j}$ (case 3.). Finally, from Assumption $(i)$, there is a finite number of $I_{j}$ and $J_{k}$ between $M_{0}$ and $M$, so that we can steer $M_{0}$ to $M$ with a finite number of such iterations, i.e., in finite time. This global strategy is illustrated in Figure 3.

Note that this proof does not provide an estimate on the minimal time $T_{0}$ needed to control. Moreover, the strategy developed here is clearly not optimal in time since it is mainly a local strategy. For instance, in Figure 3, we see that one can steer the point $I_{3} \cap \bar{J}_{3}$ directly to a point in $J_{6}$ with a single traveling wave, a strategy which is much faster than the one described in the proof. Optimizing this strategy in time seems to be an interesting open problem.

We end this section by proving a Lemma used in the Proof of Theorem 1.3. 
Lemma 3.4. Suppose that $f \in \mathcal{C}^{1}(\mathbb{R})$ satisfies (SOC+) on the interval $(L, R)$ and that $f^{\prime}(L)<$ $\sigma(L, R)$. Then, there exists $\rho_{0}>0$ such that for all $\rho \in\left(0, \rho_{0}\right)$, the function $f$ satisfies $(\mathrm{SOC}+)$ on $(L-\rho, R)$.

Proof. First, we have

$$
\left.\frac{\partial}{\partial u} \sigma(u, R)\right|_{u=L}=\frac{1}{R-L}\left(\sigma(L, R)-f^{\prime}(L)\right)>0 .
$$

Hence, there exists $\rho_{1}>0$ such that for all $\rho \in\left(0, \rho_{1}\right), \sigma(L-\rho, R)<\sigma(L, R)$. Since (SOC+) reads $\sigma(L, R)<\sigma(u, R)$ for all $u \in(L, R)$, we now have

$$
\sigma(L-\rho, R)<\sigma(u, R), \quad \text { for all } \rho \in\left(0, \rho_{1}\right), u \in(L, R) .
$$

Second, the assumption $f^{\prime}(L)<\sigma(L, R)$ together with the continuity of $f^{\prime}$ and $\sigma(\cdot, R)$ gives the existence of $\rho_{2}>0$ such that for all $\rho \in\left(0, \rho_{2}\right)$ and all $v \in(L-\rho, L)$, we have $f^{\prime}(v)<\sigma(L-\rho, R)$. Integrating this inequality with respect to $v$ on $(L-\rho, u)$, for $u \in(L-\rho, L)$ and dividing by $(u-(L-\rho))$ yields $\sigma(L-\rho, u)<\sigma(L-\rho, R)$. This is equivalent to having

$$
\sigma(L-\rho, R)<\sigma(u, R), \quad \text { for all } \rho \in\left(0, \rho_{2}\right), u \in(L-\rho, L) .
$$

Finally, setting $\rho_{0}=\min \left\{\rho_{1}, \rho_{2}\right\}$ and combining (76) and (77) concludes the proof of the lemma.

\section{Appendix: parabolic regularity estimates}

\subsection{Parabolic regularity estimates for classical solutions}

For the sake of completeness, we recall here a classical regularity result for the heat equation, that is used in the previous sections. However, we give a precise dependence with respect to $\varepsilon$, and a simple proof. Let $\Omega$ be a bounded domain of $\mathbb{R}^{n}$ with smooth boundary. For $m \geq 0$, we denote by $\mathcal{H}^{m}=\mathcal{D}\left((-\Delta)^{\frac{m}{2}}\right)$ the domain of the fractional Laplace-Dirichlet operator on $L^{2}(\Omega)$, and $\mathcal{H}^{-m}=$ $\left(\mathcal{H}^{m}\right)^{\prime}$. Here the duality is taken with respect to $\mathcal{H}^{0}=L^{2}(\Omega)$. For instance we have $\mathcal{H}^{1}=H_{0}^{1}(\Omega)$, $\mathcal{H}^{2}=H^{2}(\Omega) \cap H_{0}^{1}(\Omega)$ and $\mathcal{H}^{-1}=H^{-1}(\Omega)=\left(H_{0}^{1}(\Omega)\right)^{\prime}$. We have the following regularity result.

Lemma 4.1. Let $u(t, x)$ be a classical solution of

$$
\begin{cases}\partial_{t} u-\varepsilon \Delta u=f & \text { in }\left(0, t^{*}\right) \times \Omega, \\ u=0 & \text { on }\left(0, t^{*}\right) \times \partial \Omega, \\ \left.u\right|_{t=0}=u_{0} & \text { in } \Omega .\end{cases}
$$

and let $m \in \mathbb{R}$. Suppose that $u_{0} \in \mathcal{H}^{m}$ and $f \in L^{2}\left(0, t^{*} ; \mathcal{H}^{m-1}\right)$, then

$$
u \in \mathcal{C}^{0}\left(0, t^{*} ; \mathcal{H}^{m}\right) \cap L^{2}\left(0, t^{*} ; \mathcal{H}^{m+1}\right) \cap H^{1}\left(0, t^{*} ; \mathcal{H}^{m-1}\right)
$$

and we have, for all $t^{*}>0$,

$$
\varepsilon\left\|u\left(t^{*}\right)\right\|_{\mathcal{H}^{m}}^{2}+\varepsilon^{2} \int_{0}^{t^{*}}\|u(t)\|_{\mathcal{H}^{m+1}}^{2} d t+\int_{0}^{t^{*}}\left\|u_{t}(t)\right\|_{\mathcal{H}^{m-1}}^{2} d t=\varepsilon\left\|u_{0}\right\|_{\mathcal{H}^{m}}^{2}+\int_{0}^{t^{*}}\|f(t)\|_{\mathcal{H}^{m-1}}^{2} d t .
$$

Proof. We denote by $\left(\omega_{j}^{2}, \phi_{j}\right)_{j \in \mathbb{N}^{*}}$ the (positive) eigenvalues and associated eigenfunctions of the Laplace-Dirichlet operator on $\Omega$, forming a Hilbert basis of $L^{2}(\Omega)$. We have $-\Delta \phi_{j}=\omega_{j}^{2} \phi_{j}$ and $\left.\phi_{j}\right|_{\partial \Omega}=0$. We here suppose that $m \geq 1$. In the case $m \leq 1$, we do the following computations with smooth functions $f$ and $u$, and conclude with a density argument. Taking the inner product of (78) with $\phi_{j}$, we obtain

$$
\left\{\begin{array}{l}
u_{j, t}+\varepsilon \omega_{j}^{2} u_{j}=f_{j} \text { in }\left(0, t^{*}\right), \\
\left.u_{j}\right|_{t=0}=u_{j, 0}
\end{array}\right.
$$

where $u_{j}=\left(u, \phi_{j}\right)_{L^{2}}, u_{j, 0}=\left(u_{0}, \phi_{j}\right)_{L^{2}}$, and $f_{j}=\left(f, \phi_{j}\right)_{L^{2}}$. Multiplying the first equation by $\omega_{j}^{m-1}$ and squaring it, we have

$$
\omega_{j}^{2 m-2}\left|u_{j, t}\right|^{2}+\varepsilon^{2} \omega_{j}^{2 m+2}\left|u_{j}\right|^{2}+2 \varepsilon \omega_{j}^{2 m} u_{j} u_{j, t}=\omega_{j}^{2 m-2}\left|f_{j}\right|^{2} .
$$


Integrating this equation on $\left(0, t^{*}\right)$, we now obtain

$$
\omega_{j}^{2 m-2} \int_{0}^{t^{*}}\left|u_{j, t}\right|^{2} d t+\varepsilon^{2} \omega_{j}^{2 m+2} \int_{0}^{t^{*}}\left|u_{j}\right|^{2} d t+\varepsilon \omega_{j}^{2 m}\left|u_{j}\right|^{2}\left(t^{*}\right)=\varepsilon \omega_{j}^{2 m}\left|u_{j, 0}\right|^{2}+\omega_{j}^{2 m-2} \int_{0}^{t^{*}}\left|f_{j}\right|^{2} d t .
$$

The right hand-side is summable over $j \in \mathbb{N}^{*}$ since we supposed $\sum_{j \in \mathbb{N}^{*}} \omega_{j}^{2 m}\left|u_{j, 0}\right|^{2}=\left\|u_{0}\right\|_{\mathcal{H}^{m}}^{2}<+\infty$ and $\sum_{j \in \mathbb{N}^{*}} \omega_{j}^{2 m-2} \int_{0}^{t^{*}}\left|f_{j}\right|^{2} d t=\|f(t)\|_{L^{2}\left(0, t^{*} ; \mathcal{H}^{m-1}\right)}^{2}<+\infty$. Hence, the left hand-side is summable. Since its three terms are non-negative, they are all summable. Finally, summing (80) over $j \in \mathbb{N}^{*}$ yields (79) and the lemma is proved.

\subsection{Well-posedness of an initial-boundary value problem with low regu- larity}

We here prove the well-posedness of Problem (53) in some energy space and give the associated regularity estimates used in (68). More precisely, we consider the problem

$$
\begin{cases}u_{t}-\varepsilon u_{x x}+[W(t, x) u]_{x}=0 & \text { in }(0, T) \times(a, b) \\ \left.u\right|_{x=a}=g(t),\left.\quad u\right|_{x=b}=0 & \text { in }(0, T) \\ \left.u\right|_{t=0}=u_{0} & \text { in }(a, b)\end{cases}
$$

with $W$ and $g$ having low regularity and show that (81) is well-posed in a weak sense, viz. in the sense of transposition solutions. We have the following result.

Lemma 4.2. Suppose that $W \in L^{\infty}\left(0, T ; L^{\infty}(a, b)\right) \cap L^{1}\left(0, T ; W^{1, \infty}(a, b)\right)$ and $u_{0} \in L^{2}(a, b)$. Then there exists a unique $u \in L^{2}\left(0, T ; L^{2}(a, b)\right)$ such that

$$
\int_{0}^{T} \int_{a}^{b} u(t, x) F(t, x) d x d t=\int_{a}^{b} u_{0}(x) \varphi(0, x) d x+\varepsilon \int_{0}^{T} g(t) \varphi_{x}(t, a) d t
$$

for every test function $F \in L^{2}\left(0, T ; L^{2}(a, b)\right)$ and associated $\varphi$ satisfying

$$
\begin{cases}-\varphi_{t}-\varepsilon \varphi_{x x}-W(t, x) \varphi_{x}=F(t, x) & \text { in }(0, T) \times(a, b) \\ \left.\varphi\right|_{x=a}=0,\left.\quad \varphi\right|_{x=b}=0 & \text { in }(0, T) \\ \left.\varphi\right|_{t=T}=0 & \text { in }(a, b)\end{cases}
$$

in the classical sense. Moreover, there exists $C=C\left(T,\|W\|_{L^{\infty} L^{\infty}},\left\|W_{x}\right\|_{L^{1} L^{\infty}}\right)>0$ independent from $\varepsilon$ such that

$$
\|u\|_{L^{2}\left(0, T ; L^{2}(a, b)\right)} \leq \frac{C}{\sqrt{\varepsilon}}\left(\|g\|_{L^{2}(0, T)}+\left\|u_{0}\right\|_{L^{2}(a, b)}\right) .
$$

The solution $u$ is called the transposition solution of (81). Note that $u$ is in particular a solution of the first equation of (81) in the sense of distributions.

Proof. First notice that for any $W \in L^{\infty}\left(0, T ; L^{\infty}(a, b)\right) \cap L^{1}\left(0, T ; W^{1, \infty}(a, b)\right)$ and $F \in L^{2}\left(0, T ; L^{2}(a, b)\right)$, the backward problem (83) is well-posed in the classical sense and a regularity estimate for its solution $\varphi \in H^{1}\left(0, T ; L^{2}(a, b)\right) \cap L^{2}\left(0, T ; H^{2} \cap H_{0}^{1}(a, b)\right)$ is given in Lemma 4.3 below. We now remark that, given $u_{0} \in L^{2}(a, b)$ and $g \in L^{2}(0, T)$, the mapping

$$
l: F \mapsto \int_{a}^{b} u_{0}(x) \varphi(0, x) d x+\varepsilon \int_{0}^{T} g(t) \varphi_{x}(t, a) d t
$$

is linear. Furthermore, we have, using (85) below,

$$
\begin{aligned}
|l(F)| & \leq\left\|u_{0}\right\|_{L^{2}(a, b)}\|\varphi(0, \cdot)\|_{L^{2}(a, b)}+\varepsilon\|g\|_{L^{2}(0, T)}\left\|\varphi_{x}(\cdot, a)\right\|_{L^{2}(0, T)} \\
& \leq\left\|u_{0}\right\|_{L^{2}(a, b)}\|\varphi\|_{H^{1}\left(0, T ; L^{2}(a, b)\right)}+\varepsilon\|g\|_{L^{2}(0, T)}\|\varphi\|_{L^{2}\left(0, T ; H^{2}(a, b)\right)} \\
& \leq \frac{C}{\sqrt{\varepsilon}}\left(\left\|u_{0}\right\|_{L^{2}(a, b)}+\|g\|_{L^{2}(0, T)}\right)\|F\|_{L^{2} L^{2}}
\end{aligned}
$$


As a consequence $l$ is a continuous linear form on $L^{2} L^{2}$, and Riesz representation Theorem gives the existence of a unique $u \in L^{2} L^{2}$ satisfying (82) for every $F \in L^{2} L^{2}$. In addition, we have

$$
\|u\|_{L^{2} L^{2}} \leq \frac{C}{\sqrt{\varepsilon}}\left(\left\|u_{0}\right\|_{L^{2}(a, b)}+\|g\|_{L^{2}(0, T)}\right)
$$

and the lemma is proved.

Note that refining our estimates, we could have proved that $u \in L^{2}\left(0, T ; H^{\frac{1}{2}-\delta}(a, b)\right)$ for any $\delta>0$ (which we do not need here).

Lemma 4.3. Suppose that $W \in L^{\infty}\left(0, T ; L^{\infty}(a, b)\right) \cap L^{1}\left(0, T ; W^{1, \infty}(a, b)\right), F \in L^{2}\left(0, T ; L^{2}(a, b)\right)$ and that $\varphi$ is a solution of the backward problem (83). Then $\varphi \in L^{\infty}\left(0, T ; H_{0}^{1}\right) \cap L^{2}\left(0, T ; H^{2} \cap H_{0}^{1}\right) \cap$ $H^{1}\left(0, T ; L^{2}\right)$ and we have for some $C=C\left(T,\|W\|_{L^{\infty} L^{\infty}},\left\|W_{x}\right\|_{L^{1} L^{\infty}}\right)$, independent from $\varepsilon$,

$$
\varepsilon^{2}\|\varphi\|_{L^{\infty}\left(0, T ; H_{0}^{1}\right)}^{2}+\varepsilon^{3}\|\varphi\|_{L^{2}\left(0, T ; H^{2} \cap H_{0}^{1}\right)}^{2}+\varepsilon\left\|\varphi_{t}\right\|_{L^{2}\left(0, T ; L^{2}\right)}^{2} \leq C\|F\|_{L^{2}\left(0, T ; L^{2}\right)}^{2} .
$$

Proof. Multiplying the first line of (83) by $\varphi$ and integrating on $(a, b)$ yields

$$
-\frac{1}{2} \frac{d}{d t}\|\varphi(t, \cdot)\|_{L^{2}}^{2}+\varepsilon\left\|\varphi_{x}(t, \cdot)\right\|_{L^{2}}^{2}-\int_{a}^{b} W(t, x)\left(\frac{\varphi(t, x)^{2}}{2}\right)_{x} d x=\int_{a}^{b} F(t, x) \varphi(t, x) d x,
$$

which, after an integration by parts, gives the estimate

$$
-\frac{1}{2} \frac{d}{d t}\|\varphi(t, \cdot)\|_{L^{2}}^{2}+\varepsilon\left\|\varphi_{x}(t, \cdot)\right\|_{L^{2}}^{2} \leq \frac{1}{2}\|F(t, \cdot)\|_{L^{2}}^{2}+\frac{1}{2}\|\varphi(t, \cdot)\|_{L^{2}}^{2}+\left\|W_{x}(t, \cdot)\right\|_{L^{\infty}}\|\varphi(t, \cdot)\|_{L^{2}}^{2} .
$$

Forgetting the term $\varepsilon\left\|\varphi_{x}(t, \cdot)\right\|_{L^{2}}^{2}$ and applying Gronwall's lemma backward in time yields

$$
\|\varphi\|_{L^{\infty}\left(0, T ; L^{2}\right)}^{2} \leq \frac{1}{2} \exp \left(\left\|W_{x}\right\|_{L^{1}\left(0, T ; L^{\infty}\right)}+\frac{T}{2}\right)\|F(t, \cdot)\|_{L^{2}\left(0, T ; L^{2}\right)}^{2},
$$

since $\left.\varphi\right|_{t=T}=0$. Now integrating $(86)$ on $(0, T)$ and using (87), we obtain

$$
\varepsilon\left\|\varphi_{x}\right\|_{L^{2}\left(0, T ; L^{2}\right)}^{2} \leq\left[\frac{1}{2}+\left(\frac{T}{4}+\frac{\left\|W_{x}\right\|_{L^{1}\left(0, T ; L^{\infty}\right)}}{2}\right) \exp \left(\left\|W_{x}\right\|_{L^{1}\left(0, T ; L^{\infty}\right)}+\frac{T}{2}\right)\right]\|F\|_{L^{2}\left(0, T ; L^{2}\right)}^{2} .
$$

Next, we write the first equation of (83) as

$$
-\varphi_{t}-\varepsilon \varphi_{x x}=W \varphi_{x}+F,
$$

where the right handside is in $L^{2}\left(0, T ; L^{2}(a, b)\right)$ since $W \in L^{\infty}\left(0, T ; L^{\infty}(a, b)\right)$ and $\varphi_{x} \in L^{2}\left(0, T ; L^{2}(a, b)\right)$. The regularity estimate (79) of Lemma 4.1 for the heat equation (taken backward in time) for $m=1$ directly yields Estimate (85) and concludes the proof of the lemma.

\section{References}

[1] F. Ancona and A. Marson. On the attainable set for scalar nonlinear conservation laws with boundary control. SIAM J. Control Optim., 36:290-312, 1998.

[2] C. Bardos, A.Y. le Roux, and J.-C. Nédélec. First order quasilinear equations with boundary conditions. Comm. Partial Differential Equations, 4:1017-1034, 1979.

[3] A.M. Bruckner and K.M. Garg. The level structure of a residual set of continuous functions. Trans. Amer. Math. Soc., 232:307-321, 1977.

[4] M. Chapouly. Global controllability of nonviscous and viscous Burgers-type equations. SIAM J. Control Optim., 48:1567-1599, 2009.

[5] M. Chapouly. On the global null controllability of a Navier-Stokes system with Navier slip boundary conditions. J. Differential Equations, 247:2094-2123, 2009. 
[6] J.-M. Coron. On the controllability of the 2-D incompressible Navier-Stokes equations with the Navier slip boundary conditions. ESAIM Control Optim. Calc. Var., 1:35-75, 1996.

[7] J.-M. Coron. Some open problems on the control of nonlinear partial differential equations. In Perspectives in Nonlinear Partial Differential Equations: In honor of Haim Brezis, H. Berestycki, M. Bertsch, B. Peletier, and L. Véron, editors, volume 446 of Contemp. Math., pages 215-243. Providence, RI, 2007.

[8] J.-M. Coron and A.V. Fursikov. Global exact controllability of the 2-D Navier-Stokes equations on a manifold without boundary. Russian J. Math. Phys., 4:429-448, 1996.

[9] J.-M. Coron and S. Guerrero. Singular optimal control: A linear 1-D parabolic-hyperbolic example. Asympt. Anal., 44:237-257, 2005.

[10] C.M. Dafermos. Hyperbolic conservation laws in continuum physics. Springer-Verlag, Berlin, 2000.

[11] R. Danchin. Poches de tourbillon visqueuses. J. Math. Pures Appl., 76:609-647, 1997.

[12] L.C. Evans. Partial differential equations. Graduate Studies in Mathematics. American Mathematical Society, Providence, RI, 1998.

[13] A. Fursikov and O.Yu. Imanuvilov. Controllability of evolution equations. Lecture Notes Series. Seoul National University, Research Institute of Mathematics, Global Analysis Research Center, 1996.

[14] O. Glass. A complex-analytic approach to the problem of uniform controllability of a transport equation in the vanishing viscosity limit. J. Funct. Anal., 258:852-868, 2010.

[15] O. Glass and S. Guerrero. On the uniform controllability of the Burgers equation. SIAM J. Control Optim., 46:1211-1238, 2007.

[16] O. Glass and S. Guerrero. Some exact controllability results for the linear KdV equation and uniform controllability in the zero-dispersion limit. Asymptot. Anal., 60:61-100, 2008.

[17] O. Glass and S. Guerrero. Uniform controllability of a transport equation in zero diffusion-dispersion limit. Math. Models Methods Appl. Sci., 19:1567-1601, 2009.

[18] S. Guerrero and O.Yu. Imanuvilov. Remarks on global controllability for the Burgers equation with two control forces. Ann. Inst. H. Poincaré Anal. Non Linaire, 24:897-906, 2007.

[19] S. Guerrero and G. Lebeau. Singular optimal control for a transport-diffusion equation. Comm. Partial Differential Equations, 32:1813-1836, 2007.

[20] T. Horsin. On the controllability of the Burgers equation. ESAIM Control Optim. Calc. Var., 3:83-95, 1998.

[21] S.N. Kruzkov. First order quasilinear equations with several independent variables. (russian). Mat. Sb. (N.S. ), 81:228-255, 1970.

[22] J.-L. Lions. Contrôlabilité exacte et stabilisation de systèmes distribués. Tome 2. Perturbations. Collection RMA. Masson, Paris.

[23] D.R. Smart. Fixed point theorems. Cambridge University Press, London-New York, 1974. 